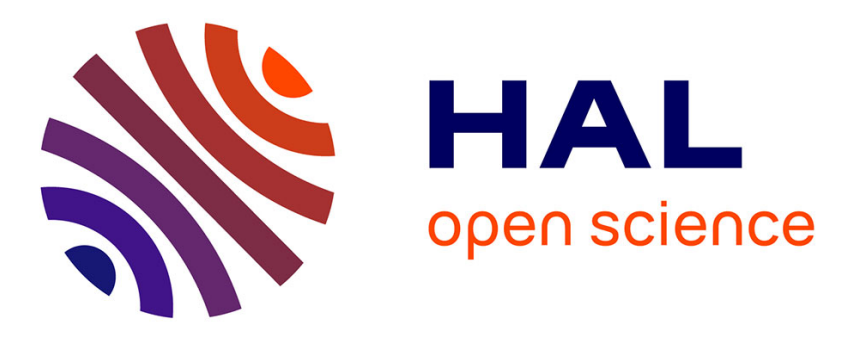

\title{
Oriented basement membrane fibrils provide a memory for F-actin planar polarization via the Dystrophin-Dystroglycan complex during tissue elongation
}

Fabiana Cerqueira Campos, Cynthia Dennis, Hervé Alégot, Cornelia Fritsch, Adam Isabella, Pierre Pouchin, Olivier Bardot, Sally Horne-Badovinac, Vincent Mirouse

\section{- To cite this version:}

Fabiana Cerqueira Campos, Cynthia Dennis, Hervé Alégot, Cornelia Fritsch, Adam Isabella, et al. Oriented basement membrane fibrils provide a memory for F-actin planar polarization via the Dystrophin-Dystroglycan complex during tissue elongation. Development (Cambridge, England), 2020, 147 (7), pp.dev186957. 10.1242/dev.186957 . hal-03009513

\section{HAL Id: hal-03009513 https://hal.science/hal-03009513}

Submitted on 20 Nov 2020

HAL is a multi-disciplinary open access archive for the deposit and dissemination of scientific research documents, whether they are published or not. The documents may come from teaching and research institutions in France or abroad, or from public or private research centers.
L'archive ouverte pluridisciplinaire HAL, est destinée au dépôt et à la diffusion de documents scientifiques de niveau recherche, publiés ou non, émanant des établissements d'enseignement et de recherche français ou étrangers, des laboratoires publics ou privés. 


\title{
Oriented basement membrane fibrils provide a memory for F-actin planar polarization via the Dystrophin-Dystroglycan complex during tissue elongation
}

\author{
Fabiana Cerqueira Campos ${ }^{1, *}$, Cynthia Dennis ${ }^{1}$, Hervé Alégot ${ }^{1, \pm}$, Cornelia Fritsch $^{1, \S}$, Adam Isabella ${ }^{2, \pi}$, \\ Pierre Pouchin ${ }^{1}$, Olivier Bardot ${ }^{1}$, Sally Horne-Badovinac ${ }^{2}$ and Vincent Mirouse ${ }^{1, * *}$
}

\begin{abstract}
How extracellular matrix contributes to tissue morphogenesis is still an open question. In the Drosophila ovarian follicle, it has been proposed that after Fat2-dependent planar polarization of the follicle cell basal domain, oriented basement membrane (BM) fibrils and F-actin stress fibers constrain follicle growth, promoting its axial elongation. However, the relationship between BM fibrils and stress fibers and their respective impact on elongation are unclear. We found that Dystroglycan (Dg) and Dystrophin (Dys) are involved in BM fibril deposition. Moreover, they also orient stress fibers, by acting locally and in parallel to Fat2. Importantly, Dg-Dys complexmediated cell-autonomous control of $\mathrm{F}$-actin fiber orientation relies on the preceding BM fibril deposition, indicating two distinct but interdependent functions. Thus, the Dg-Dys complex works as a crucial organizer of the epithelial basal domain, regulating both F-actin and BM. Furthermore, BM fibrils act as a persistent cue for the orientation of stress fibers that are the main effector of elongation.
\end{abstract}

KEY WORDS: Morphogenesis, Extracellular matrix, Drosophila, Tissue elongation, Planar cell polarity, Dystrophin, Dystroglycan, Basement membrane

\section{INTRODUCTION}

Deciphering the mechanisms underlying tissue morphogenesis is crucial for our fundamental understanding of development and also for regenerative medicine. Building organs generally requires the precise modeling of a basement membrane extracellular matrix (ECM), which in turn can influence tissue shape (Dzamba and DeSimone, 2018; Sekiguchi and Yamada, 2018; Isabella and Horne-Badovinac, 2015a,b). However, the mechanisms driving the assembly of a specific basement membrane (BM) and how this BM then feeds forward on morphogenesis are still poorly understood.

\footnotetext{
${ }^{1}$ iGReD (Institute of Genetics, Reproduction and Development), Université Clermont Auvergne - UMR CNRS 6293 - INSERM U1103, Faculté de Médecine, 28 Place Henri-Dunant, 63000 Clermont-Ferrand, France. ${ }^{2}$ Committee on Development, Regeneration and Stem Cell Biology, and Department of Molecular Genetics and Cell Biology - The University of Chicago, 920 East 58th Street, Chicago IL 60653, USA.

*Present address: Institut Pasteur, Department of Developmental and Stem Cell Biology/CNRS UMR3738, 25 rue du Dr. Roux 75015, Paris. ¥Present address: IBDM, Parc Scientifique de Luminy, 163 Avenue de Luminy, 13009, Marseille, France. \$Present address: University of Fribourg, Department of Biology, Chemin du musée 10, 1700 Fribourg, Switzerland. TPresent address: Division of Basic Sciences, Fred Hutchinson Cancer Research Center, 1100 Fairview Avenue North, Seattle WA 98109, USA.

**Author for correspondence (vincent.mirouse@uca.fr)
}

(D) O.B., 0000-0001-6561-1361; V.M., 0000-0001-5823-342X

Received 2 December 2019; Accepted 24 February 2020
Drosophila oogenesis offers one of the best tractable examples in which such a morphogenetic process can be studied. Each ovarian follicle, which is composed of a germline cyst surrounded by the somatic follicular epithelium, undergoes a dramatic growth, associated with tissue elongation, starting from a little sphere and ending with an egg in which the anteroposterior (AP) axis is 3 -fold longer than the mediolateral (ML) axis (Fig. 1A). This elongation is roughly linear from the early to the late stages, but can be separated in at least two mechanistically distinct phases (Alégot et al., 2018; Aurich and Dahmann, 2016). The first phase (from stage 3 to stage 8 ; hereby 'early stages') requires a double gradient of JAK-STAT pathway activity that emanates from each pole and that controls myosin II-dependent apical pulsations (Alégot et al., 2018). In the second phase, from stage 7-8, elongation depends on the atypical cadherin Fat2 that is part of a planar cell polarity (PCP) pathway orienting the basal domain of epithelial follicle cells (Gutzeit et al., 1991; Barlan et al., 2017; Chen et al., 2016; Viktorinová et al., 2009). Earlier during oogenesis, Fat 2 gives a chirality to the basal domain cytoskeleton in the germarium, the structure from which new follicles bud (Chen et al., 2016). This chirality is required to set up a process of oriented collective cell migration perpendicularly to the elongation axis that induces follicle revolutions from stage 1 to stage 8 (Haigo and Bilder, 2011; Chen et al., 2016; Viktorinová and Dahmann, 2013). From each migrating cell, Fat2 also induces, in the rear adjacent cell, the formation of planar-polarized protrusions that are required for rotation (Cetera et al., 2014; Barlan et al., 2017). These rotations allow the polarized deposition of BM fibrils, which involves a Rab10-dependent secretion route targeted to the lateral domain of the cells. These BM fibrils are detectable from stage 4 onwards and persist until late developmental stages (Haigo and Bilder, 2011; Isabella and Horne-Badovinac, 2016). Follicle rotation also participates in the planar cell polarization of integrin-dependent basal stress fibers that are oriented perpendicularly to the AP axis (Cetera et al., 2014). Moreover, at stage 7-8, a gradient of matrix stiffness controlled by the JAK-STAT pathway and Fat 2 contributes to elongation (Crest et al., 2017). Then, from stage 9, the epithelial cell basal domain undergoes anisotropic oscillations, as a result of periodic contraction of the oriented stress fibers, which also promotes follicle elongation (He et al., 2010; Qin et al., 2017). To explain the impact of fat 2 mutations on tissue elongation, it is generally accepted that oriented stress fibers and BM fibrils act as a molecular corset that constrains follicle growth in the ML axis and promotes its elongation along the AP axis. However, the exact contribution of F-actin versus $\mathrm{BM}$ to this corset is still unclear, as is whether the orientations of stress fibers and of BM fibrils are causally linked.

Here, we analyzed the function of Dystrophin (Dys) and Dystroglycan (Dg) during follicle elongation. Dys and Dg are the two main components of the Dystrophin-associated protein 

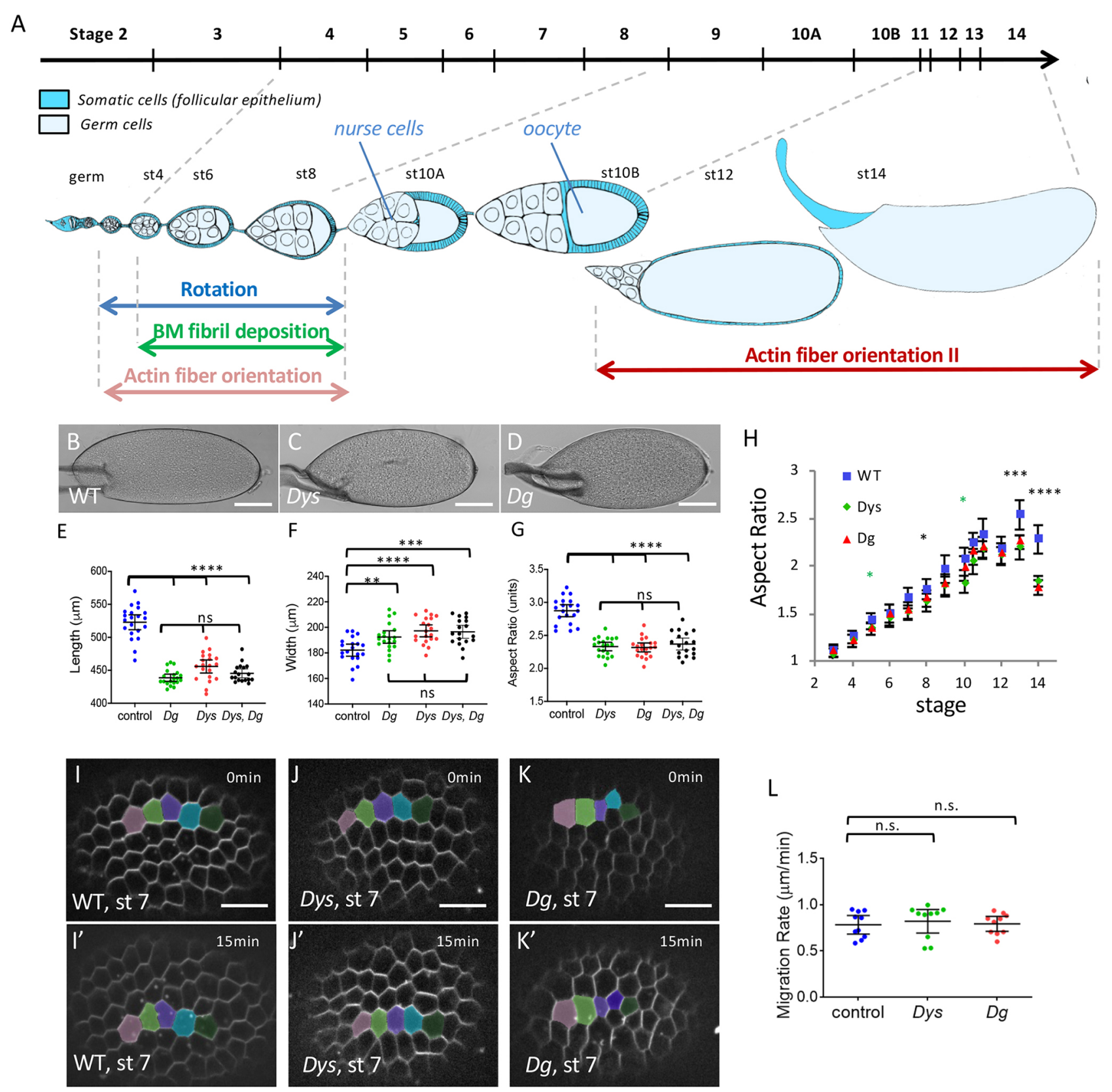

Fig. 1. The DAPC is involved in follicle elongation but not rotation. (A) Scheme of an ovariole with the main events involved in follicle elongation. The top line indicates the time scale of the different developmental stages. The ovariole is oriented anterior to posterior (germ, germarium). Each follicle is composed of a germline cyst surrounded by the follicular epithelium. Rotation occurs from very early to stage 8 . It promotes F-actin fiber orientation and allows polarized BM fibril deposition, both perpendicularly to the elongation axis. At stage 11, actin fibers lose their orientation and then progressively reorient (orientation II).

(B-D) Representative mature eggs from WT (B), Dys $\mathrm{E}^{E 17 / E x e / 6184}$ (C) and $D g^{\mathrm{O} 46 / 083}$ females (D). Scale bars: $100 \mu \mathrm{m}$. (E-G) Quantification of the length (E), the

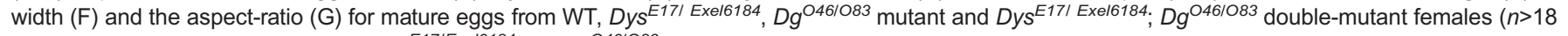
eggs). (H) Elongation kinetics of WT, Dys ${ }^{E 17 / E x e / 6184}$ and $D g^{046 / O 83}$ mutant follicles ( $n>6$ follicles for each point, green asterisks for Dys, black asterisks for Dys and $D g)$. There are two series of value for stage 10, corresponding to stage 10A and 10B. $\left(\mathrm{I}-\mathrm{K}^{\prime}\right)$ Images of rotation movies of stage $7 \mathrm{WT}\left(\mathrm{I}, \mathrm{I}^{\prime}\right)$, Dys $\mathrm{S}^{E 17 /} \mathrm{Exe} / 6184\left(\mathrm{~J}, \mathrm{~J}^{\prime}\right)$ and $D^{\mathrm{O} 46 / 083}\left(\mathrm{~K}, \mathrm{~K}^{\prime}\right)$ mutant follicles. Scale bars: $10 \mu \mathrm{m}$. (L) Rotation velocity of WT, Dys ${ }^{\text {E17/Exe/6184 }}$ and $D g^{\mathrm{O} 46 / 083}$ mutant stage 7 follicles ( $n>8$ follicles). For all panels, error bars represent s.d.; ${ }^{*} P<0.01,{ }^{* \star} P<0.005$, ${ }^{\star \star \star} P<0.001$, ${ }^{* \star \star *} P<0.0001$ (unpaired $t$-test). n.s., not significant.

complex (DAPC), an evolutionarily conserved transmembrane complex that links the ECM (via Dg) to the F-actin cytoskeleton (via Dys) (Barresi and Campbell, 2006). This complex is expressed in a large variety of tissues and is implicated in many congenital degenerative disorders. Loss-of-function studies in model organisms have revealed an important morphogenetic role for Dg during development, usually linked to defects in ECM secretion, assembly or remodeling (Clements et al., 2017; Henry and
Campbell, 1998; Satz et al., 2010; Bello et al., 2008; Buisson et al., 2014; Naegeli et al., 2017; Yatsenko and Shcherbata, 2014). A developmental role for Dys is less clear, possibly because of the existence of several paralogs in vertebrates. As Drosophila has only one $D g$ and one Dys gene, it is a promising model for their functional study during development and morphogenesis.

We found that $D g$ and Dys are required for follicle elongation and proper BM fibril formation early in fly oogenesis. During these 
early stages, DAPC loss and hypomorphic fat 2 conditions similarly delay stress fiber orientation. However, DAPC promotes this alignment more locally than Fat2. Moreover, DAPC genetically interacts with fat 2 in different tissues, suggesting that they belong to a common morphogenetic network. Later in oogenesis, Dg and Dys are required for stress fiber orientation in a cell-autonomous manner. This is the period when the main elongation defect is seen in these mutants, arguing for a more determinant role for stress fibers compared with BM fibrils in the elongation process. Nonetheless, this latter function depends on the earlier DAPC function in BM fibril deposition. We propose that $\mathrm{BM}$ fibrils serve as a $\mathrm{PCP}$ memory for the late stages that are used as a template by the DAPC for F-actin stress fiber alignment.

\section{RESULTS}

Dys and Dg mutants reveal a new phase of follicle elongation

As Drosophila follicle elongation requires many proteins that function at the basal side of the follicular epithelium, we checked whether the DAPC could be involved in this process. Both Drosophila Dg and Dys null mutants are viable and we used combinations of two different null alleles for each of them (Christoforou et al., 2008). Mature eggs from mutant females are significantly shorter and wider (Fig. 1B-F), with an aspect ratio (AR) around 2.3 compared with 2.9 in wild-type (WT) follicles (Fig. 1G). Importantly, such a phenotype can be rescued by restoring expression in the follicle cells, confirming that it is associated with these mutations, and indicating that the DAPC is required for elongation in the follicle cells (see below). Moreover, double-mutant eggs for Dys and $D g$ show the same defect as single mutants, confirming that these genes work together for follicle elongation (Fig. 1E-G). Comparison of the elongation kinetics in mutant and WT follicles shows that such morphological differences appear mainly during the later stages of oogenesis (stages 12 to 14), although a slight, but significant defect is present around stage 8 (Fig. 1H). The Dg and Dys null mutants' elongation kinetic curves are clearly different from those of fat 2 mutant in which elongation is strongly affected from stages 7-8 (Alégot et al., 2018; Aurich and Dahmann, 2016). Thus, the DAPC is involved in follicle morphogenesis, and its specific temporal requirement suggests the existence of an elongation phase that is mechanistically different from the Fat2-dependent one.

Next, to determine how the DAPC contributes to follicle elongation, we first checked the collective cell migration that occurs during the early stages because it is upstream of many subsequent events that are potentially important for elongation. Both $D y s$ and $D g$ null mutant follicles rotate and the migration velocity is not significantly different from that of WT follicles (Fig. 1I-L). Thus, the DAPC is not involved in the collective cell migration and in the initial PCP established by Fat2, confirming that it acts through a distinct mechanism. We then focused our phenotypic analysis on the two events induced by follicle rotation: BM fibril deposition and stress fiber orientation.

\section{The DAPC is important for BM fibril deposition}

The follicle cell BM contains two ECM types: a planar BM without a specific organization visible by confocal microscopy, and BM fibrils that are oriented perpendicular to the follicle AP axis. BM fibrils are deposited during follicle rotation via a secretory route targeted to the cell lateral domain, and can be observed by monitoring the expression of the main ECM components: Collagen IV (ColIV), Perlecan (Pcan; also known as Trol) and Laminin A (LanA), the two latter being direct Dg ligands (Gutzeit et al., 1991; Haigo and Bilder, 2011; Isabella and Horne-Badovinac, 2016). ColIV and Pcan can be visualized using the
Viking- and Trol-GFP protein traps, respectively (Morin et al., 2001). Moreover, a fosmid containing the whole Laminin A gene tagged with GFP has also been generated (LanA-GFP) (Sarov et al., 2016). We also employed the MiMIC system to generate RFP-tagged Pcan (Venken et al., 2011). This line gives an identical pattern to Pcan-GFP and is homozygous viable and fertile, indicating that it does not disrupt Pcan function (Fig. S1A). Comparison of the BM fibril composition using these different tagged proteins (Fig. 2A-B") shows that in WT follicles, Pcan-RFP colocalizes exactly with ColIV-GFP and LanA-GFP. This indicates that BM fibrils are uniformly composed of these three proteins, as has been previously shown by immunostaining (Isabella and Horne-Badovinac, 2016).

We then wondered whether BM fibril deposition is affected in Dys and $D g$ mutants. In these two mutants, the general targeting of $\mathrm{BM}$ secretion towards the basal domain is normal, with no visible apical secretion as has been observed in different mutants affecting BM secretion (Fig. S1B-D) (Denef et al., 2008; Devergne et al., 2014, 2017; Lerner et al., 2013). However, images in the focal plane of the $\mathrm{BM}$ reveal a very strong decrease of the ColIV ${ }^{-}$fibrils in the Dys null mutant (Fig. 2D). This observation is also true for LanA and Pcan (Fig. 2C,E). Quantification of the BM fibril fraction on stage 8 follicles, which corresponds to the fluorescence fraction present in the fibrils, indicates that it is about $15 \%$ in the WT situation, but drops to $2 \%$ in Dys mutants, and that a similar phenotype is observed in $D g$ mutants (Fig. 2F). This defect is specific to the fibrils and does not affect unpolarized matrix because the total basal fluorescence intensity tends to increase in these mutants, although it is variable from one follicle to another (Fig. S1E). Loss of the DAPC affects both BM fibril number and length (Fig. 2G,H). As a result of this defect, some fibrils are still visible in stage 12 WT follicles but hardly detectable in Dys and Dg mutant ones (Fig. S1F-I). This shows that the DAPC is involved in BM fibril deposition.

This deposition depends on the lateral secretion of ECM proteins while the rotation facilitates both the deposition and orientation of the fibrils in the BM (Isabella and Horne-Badovinac, 2016). The lateral secretion of $\mathrm{BM}$ proteins can be visualized by immunostaining on non-permeabilized follicle cells to detect only the proteins localized between the cells. Such an approach on Dys mutant clones shows that some ColIV is detected, as observed between WT cells (Fig. 2I-J). This suggests that Dys does not directly interfere with the lateral targeting of BM fibril secretion. In a mutant blocking rotation, such as fat 2, BM proteins accumulate in the lateral space between cells, whereas no massive accumulation is observed in a Dys mutant, suggesting that the BM fibril defect associated with DAPC loss of function cannot be explained by a failure of the latest step of BM fibril deposition (Fig. 2K) (Isabella and Horne-Badovinac, 2016). Moreover, in a fat2, Dys double mutant we observed the same lateral accumulation of ECM proteins as in the fat 2 mutant, confirming that lateral secretion is not affected by DAPC loss of function (Fig. 2K,L). It is therefore unclear by which mechanism the DAPC contributes to BM fibril formation. Nonetheless, these mutants are the first examples of mutations that specifically reduce BM fibril formation without any defect in the general targeting of basal secretion or in rotation, offering a unique opportunity to address the functional relevance of the fibrils.

\section{The DAPC contributes to stress fiber orientation during early stages}

In parallel with the analysis of BM fibrils, we also studied the impact of the loss of the DAPC on basal F-actin organization. This alignment is still weak at stage 4 and then increases to reach a maximum around stage 8 (Fig. 3A-F) (Cetera et al., 2014). In both 

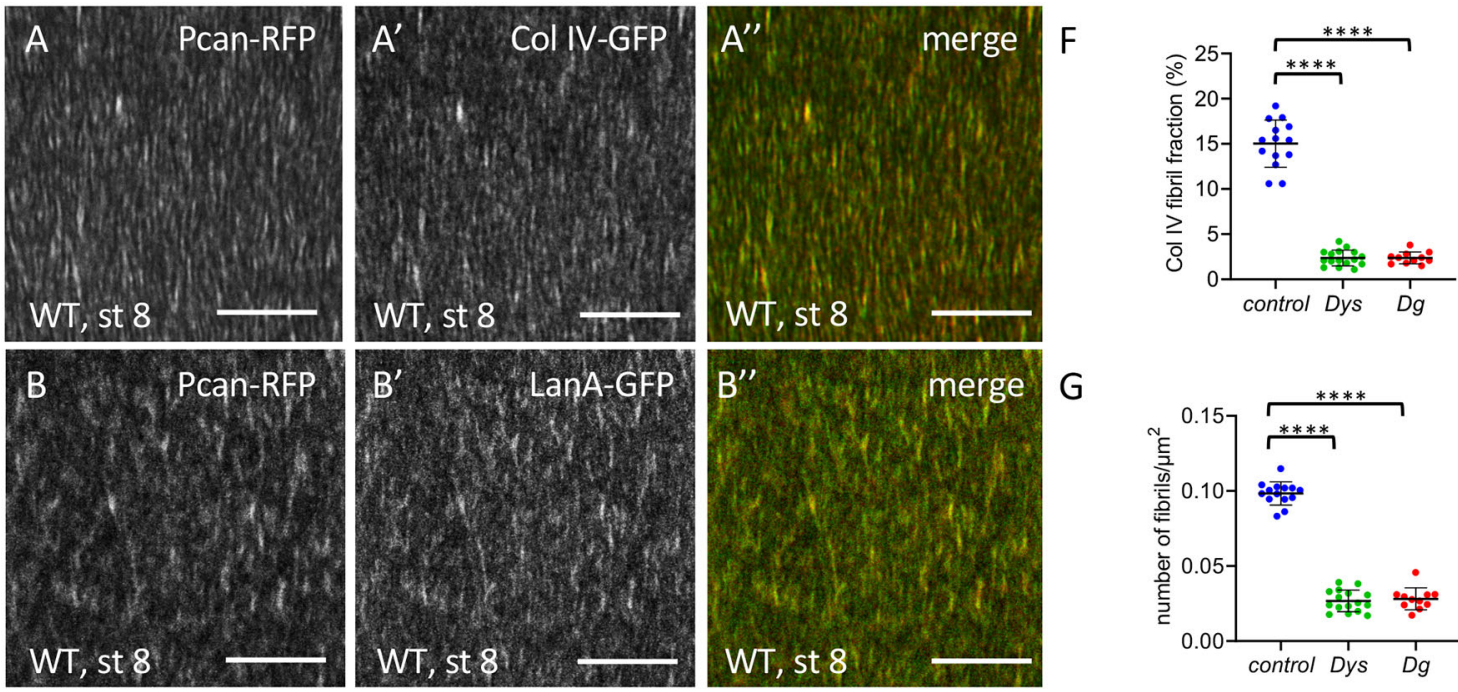

G
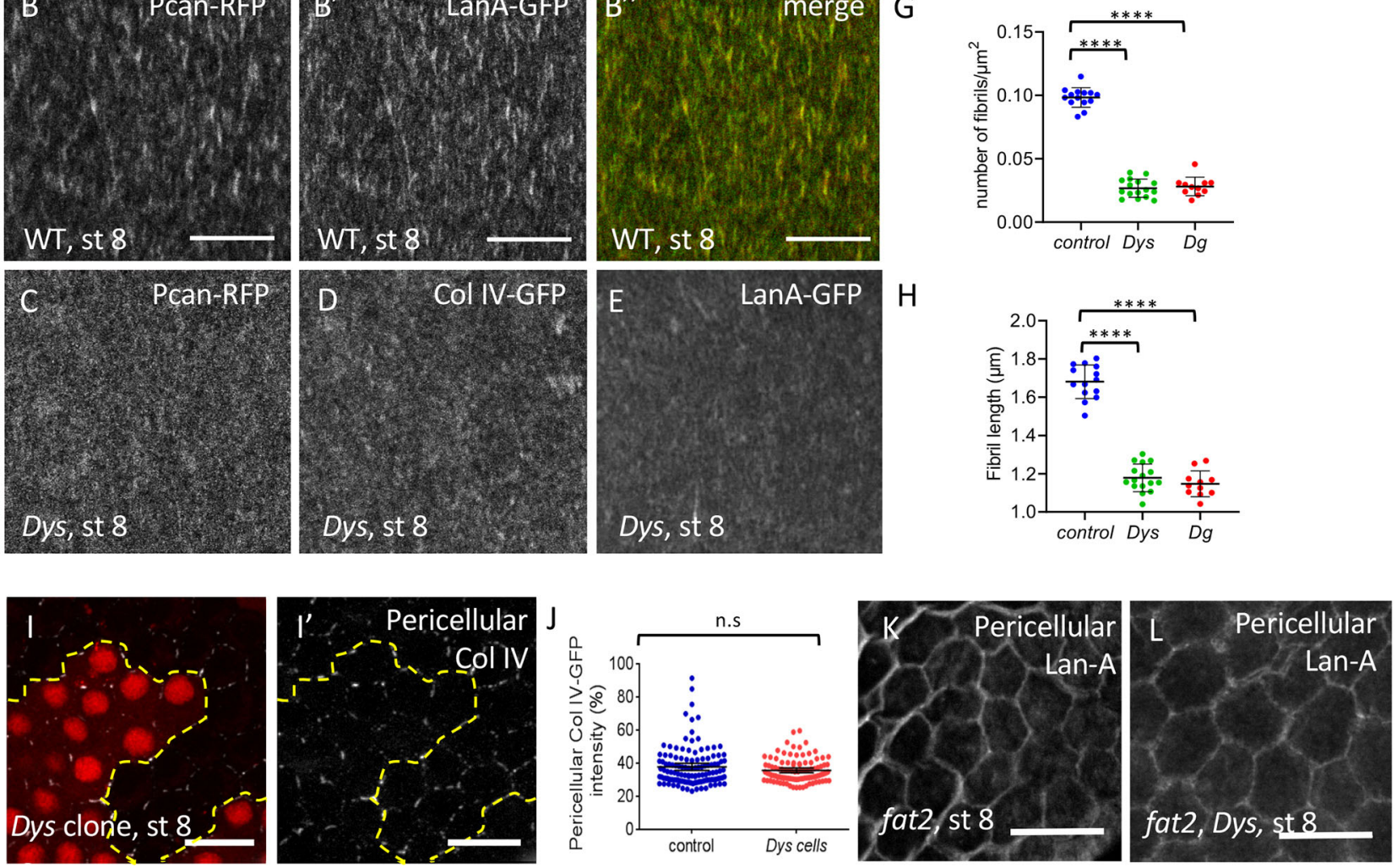

Fig. 2. The DAPC is important for BM fibril deposition. (A-B") Basal view of WT ( $w^{1118}$ ) follicle ECM at stage 8 visualized with Pcan-RFP and CollV-GFP (A)

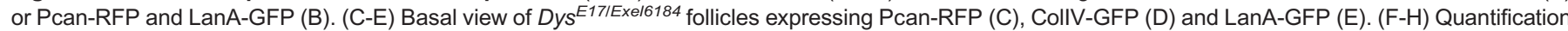
of the BM fibril fraction (F), fibril numbers $(\mathrm{G})$ and fibril length $(\mathrm{H})$ at stage 8 in WT, Dys ${ }^{\text {E17/Exe/6184 }}$ and $D g^{\mathrm{O} 86 / 043}$ mutant follicles ( $n>10$ follicles). (I,I') Mutant clones for Dys ${ }^{\text {Exel6184 }}$ marked by the absence of RFP and stained to detect pericellular CollV. Dashed line indicates the border between WT and mutant cells. (J) Quantification of pericellular CollV intensity around WT and Dys mutant cells ( $n>20$ cells). (K,L) Accumulation of pericellular LanA in fat2 (K) and fat2, Dys (L) mutant follicles. For all panels, error bars represent s.d.; ${ }^{* * *} P<0.0001$ (unpaired $t$-test). n.s., not significant. Scale bars: $10 \mu \mathrm{m}$.

Dys and Dg null mutants, although the stress fibers are globally oriented perpendicular to the AP axis in mutant follicles, quantification of the angular distribution reveals a delay in their proper orientation at stage 4 and 6 to reach a normal level at stage 8 (Fig. 3A-R). Stress fiber orientation can be also analyzed by the determination of the tissue order parameter (Tissue OP) based on a method previously described (Cetera et al., 2014). A complete random orientation Tissue $\mathrm{OP}$ value will tend to 0 , whereas a perfect alignment between the cells will tend to 1 (Fig. 3S). This method confirms that $D y s$ and $D g$ mutants behave similarly, with a delay in the proper stress fiber alignment (Fig. 3T). Although rotation is required for stress fiber orientation, this motion is not affected by DAPC loss of function. Thus, this complex likely acts downstream of or in parallel with rotation to orient stress fibers.

We also addressed whether $D y s$ is required cell-autonomously to promote stress fiber orientation by generating mutant clones and we focused on stage 5, when the defect is obvious in Dys mutant follicles. Comparing the mutant cells versus the WT ones in a same follicle, we observed no apparent effect on F-actin density but a cellautonomous effect on their orientation that can be quantified by the Tissue OP (Fig. 3U-V). Thus, Dys promotes stress fiber orientation in each cell.

\section{The DAPC and Fat2 have partially redundant functions}

We found that the DAPC has only a minor impact on the early phase of stress fiber orientation, with a delayed orientation of actin fibers that is achieved by stage 8 , suggesting alternative mechanisms to orient fibers at these stages (4-8). Similarly, deletion of the intracellular part of Fat2 (fat2-DeltaICD) induces a hypomorphic defect with only a slight delay of stress fiber orientation, despite a slower rotation (Aurich and Dahmann, 2016; Barlan et al., 2017; Chen et al., 2017). We therefore wondered what could be the relationship between these two genetic backgrounds. We generated a double-mutant background for fat2/fat2-DeltaICD and Dys null alleles (Dys, fat2-DeltaICD/Dys, fat 2 ) and compared stress fiber alignment for these different genotypes (Fig. 4A-D). Tissue OP 

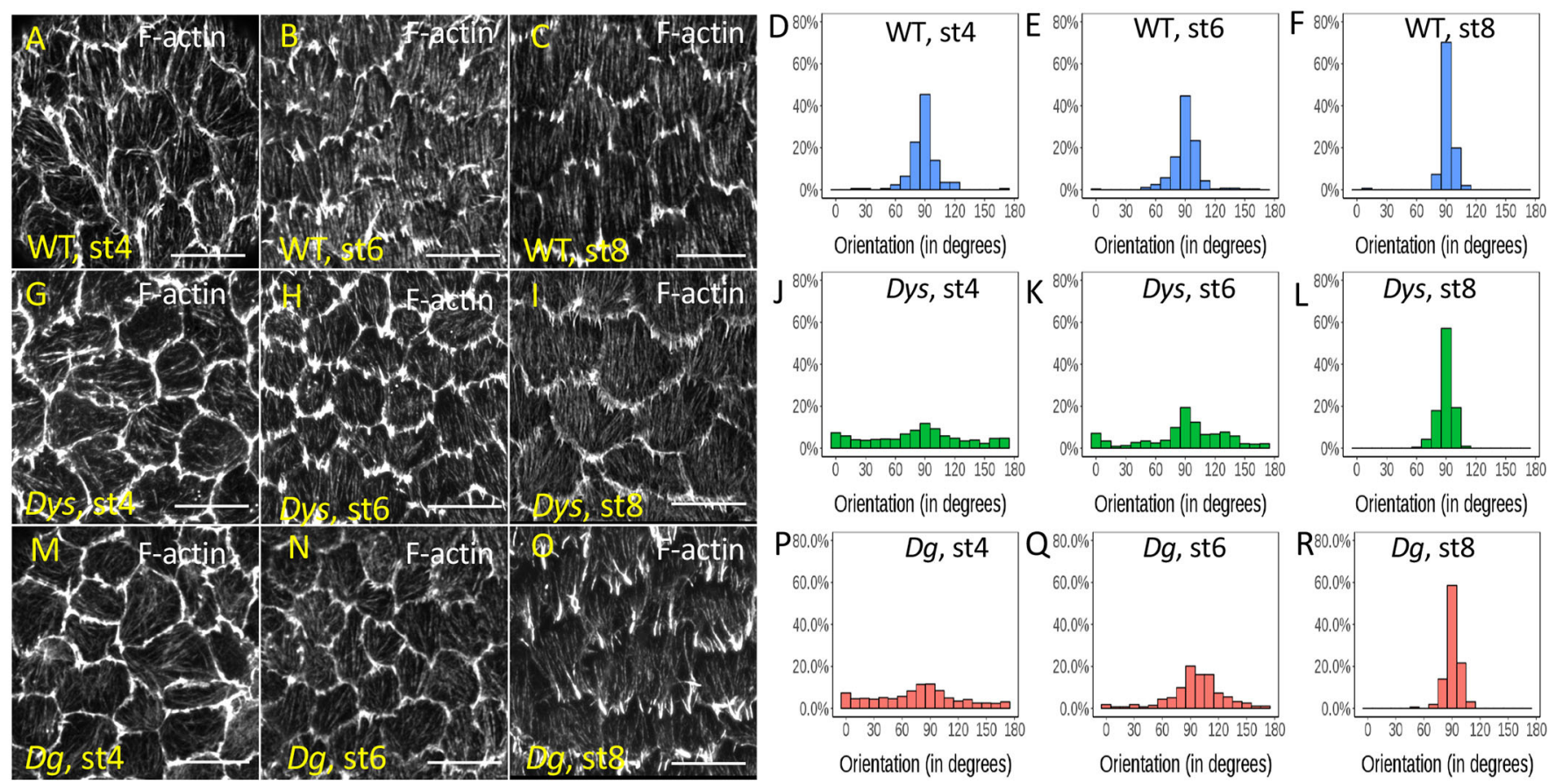

$\mathrm{S}$

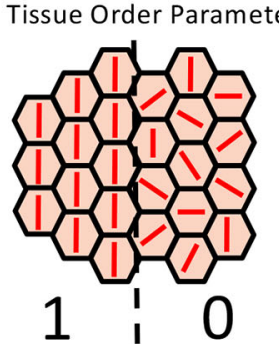

T

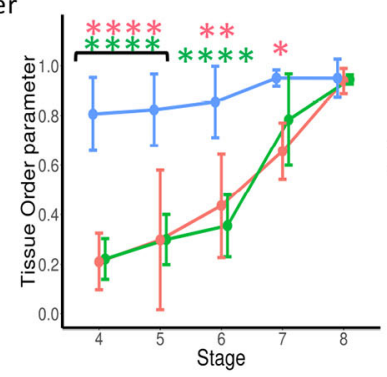

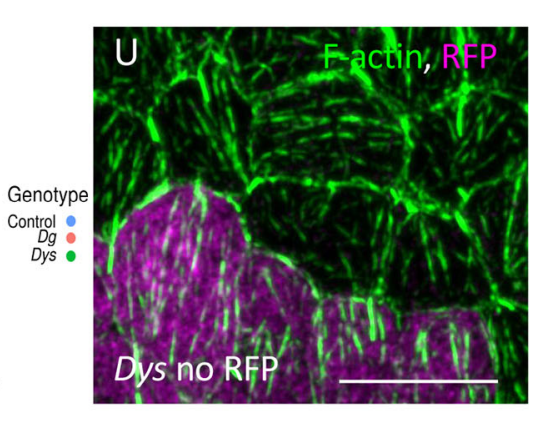

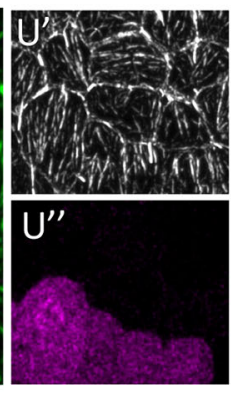

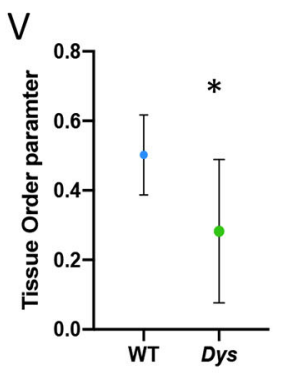

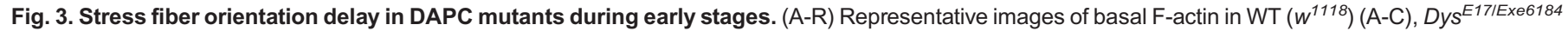
(G-I) and $\mathrm{Dg}^{\mathrm{O} 86 / \mathrm{O} 43}$ (M-O) follicles at stage 4, 6 and 8 and quantification of the corresponding angular distribution (D-F,J-L,P-R). (S) Illustration of Tissue OP: if all the cells have the same orientation Tissue OP is equal to one, if orientation is random Tissue OP is equal to zero. (T) Tissue OP analysis of control, $D y s^{E 17 / E x e / 6184}$ and Dg ${ }^{\mathrm{O} 6 / \mathrm{O} 83}$ follicles. A two-way ANOVA was performed, followed by applicable post-hoc pairwise comparisons (Tukey). (U-U") F-actin in a mutant clone for Dys ${ }^{E x e / 6184}$ marked by the absence of RFP (purple) at stage 5. Note the cell-autonomous effect on stress fiber orientation. (V) Tissue OP quantification $D y s{ }^{E x e l 6184}$ mutant clones and surrounding WT cells at stage 5 . Error bars represent s.d. ( $n>7$ follicles for each stage and each genotype). ${ }^{*}<<0.01$, ${ }^{* *} P<0.005,{ }^{* * *} P<0.0001$. Scale bars: $10 \mu \mathrm{m}$.

analysis shows that single mutants are similar and that the double mutants display an additive effect (Fig. 4E,I), an additivity that is also observed on follicle elongation (Fig. 4Q). Thus, Dys and Fat2 probably work in parallel for stress fiber orientation once Fat2 has initiated follicle rotation.

To get a better understanding of this additivity, we performed a deeper analysis of stress fiber orientation. First, we wondered whether the alignment robustness was the same in each cell and we therefore analyzed the cell order parameter (Cell OP) (Fig. 4F). This clearly shows that the mutants behave in a different manner, with fat2DeltaICD behaving similarly to WT and with Dys and Dys, fat2DeltaICD giving an identical weaker Cell OP (Fig. 4J). This effect on Cell OP of Dys can be confirmed by its analysis on Dys mutant clones (Fig. S2E). Moreover, a close observation of Dys single cells reveals more divergent stress fibers than we have observed in fat2-DeltaICD cells (Fig. S2C,D). This indicates that Dys promotes stress fiber alignment in each cell, a process that does not seem to involve Fat2. We also calculated a local order parameter (Local OP), determined for each cell on a cluster including this cell and its neighbors within a given radius (Fig. 4G). Globally, for all mutant genotypes that we considered, Local OP values are initially higher than the respective Tissue OP ones, but they tend to be equal to the Tissue OP values when the analyzed radius is increased (Fig. S2F). The additivity of Dys and fat 2 can be also observed on the Local OP (Fig. 4K, Fig. S2F). Finally, we looked at the difference between Local OP, for a radius corresponding to one row of neighbors, and Tissue OP (Fig. 4H). Interestingly, we noticed a stronger Local/Tissue OP difference for fat2-DeltaICD than for Dys mutants, a difference culminating at stage 6 , indicating that stress fiber orientation is less affected at a small scale in fat 2 than in Dys mutants (Fig. 4L, Fig. S2G). This difference can be also directly observed on images of F-actin orientation (Fig. S2A,B). For fat2-DeltaICD, several patches of neighboring cells with the same orientation but changing from one patch to another can be observed. For Dys, we do not detect these patches but do see changes in orientation from one cell to another. Altogether, these data indicate that Dys promotes stress fiber planar polarization at a cellular and local scale whereas Fat2, or at least its intracellular domain, acts at a more global scale. 


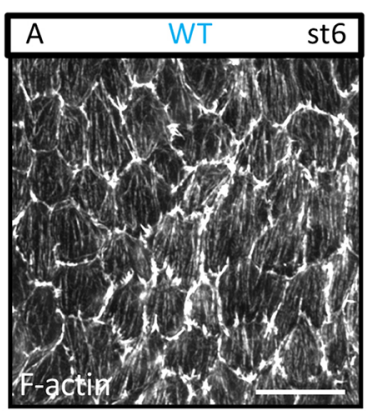

E

Tissue Order Parameter

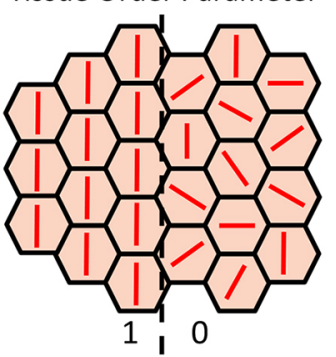

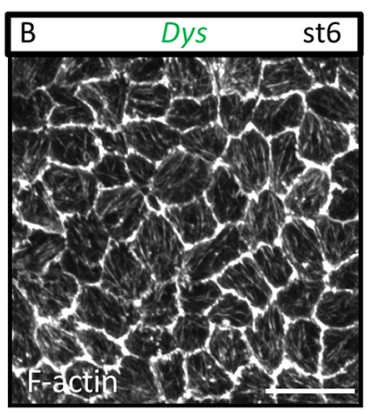

$\mathrm{F}$

Cell Order Parameter

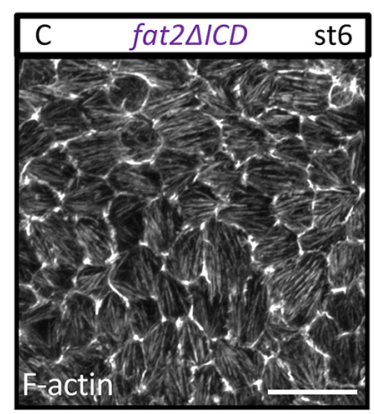

G

Local Order Parameter

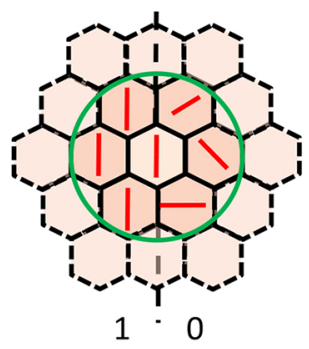

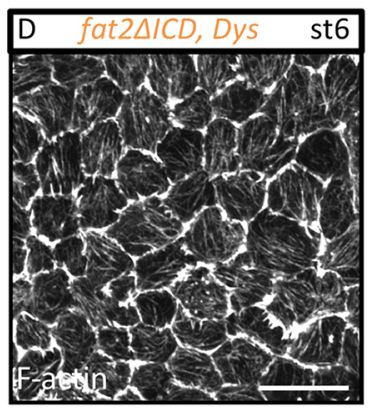

$\mathrm{H}$

Delta Local-Tissue OP
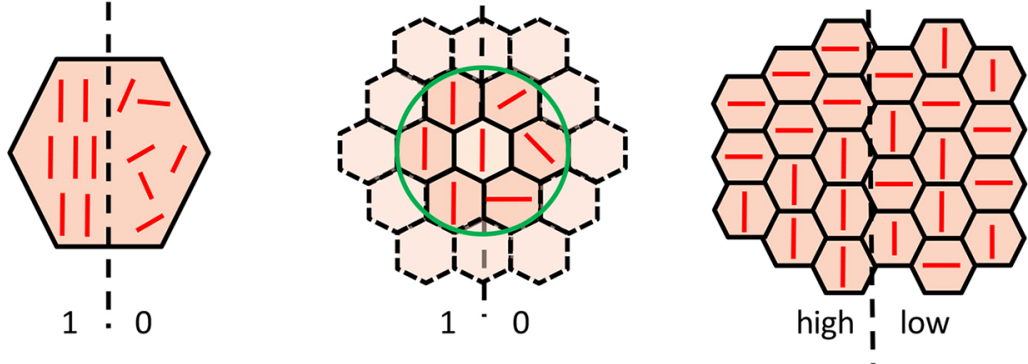

$\mathrm{L}$
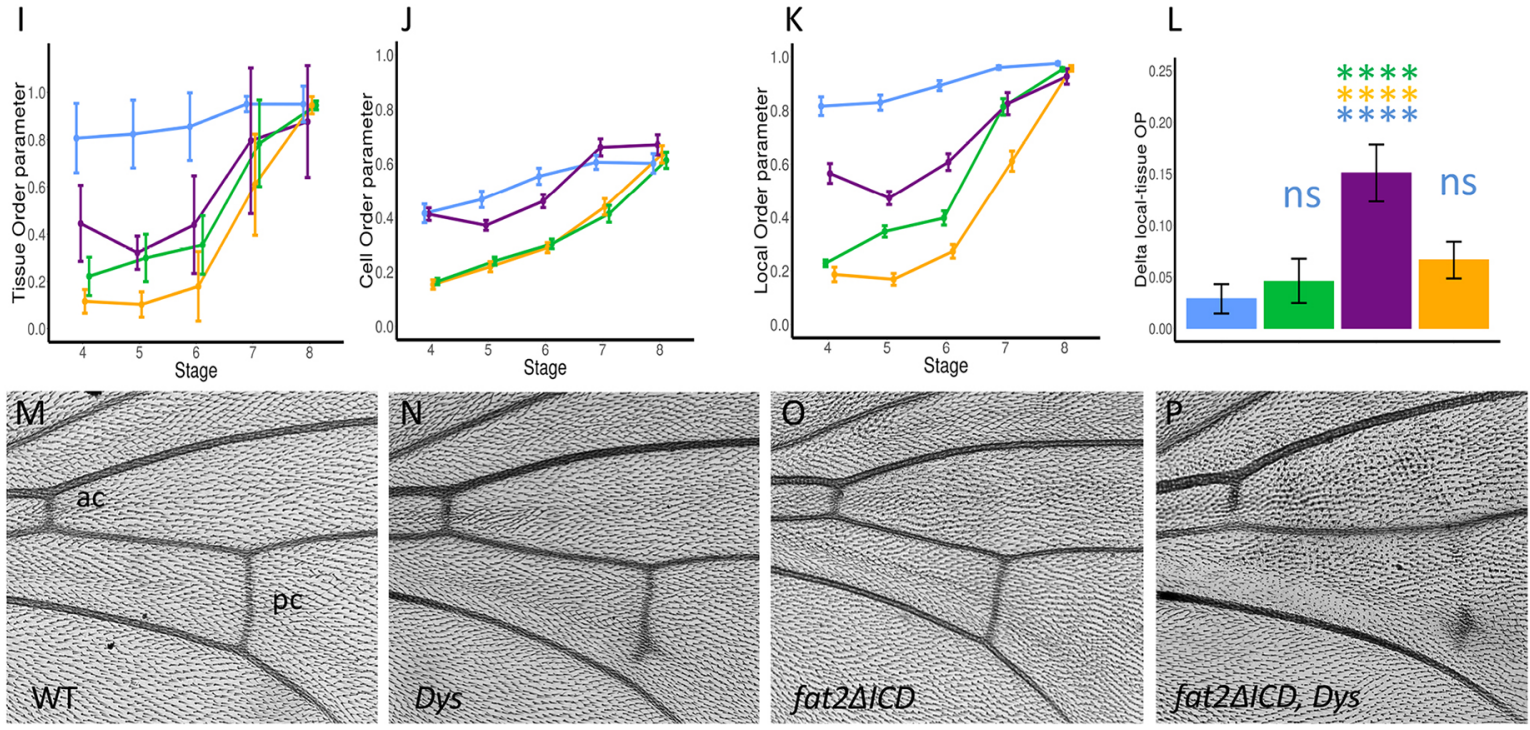

Q

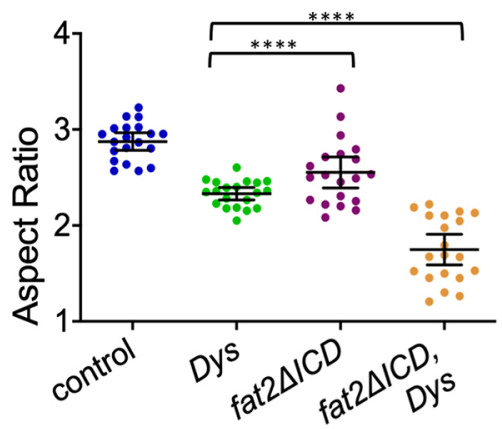

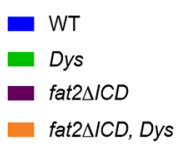

fat2 $\triangle / C D, D y s$

Fig. 4. Genetic interaction between fat2 and Dys. (A-D) Representative images of stress fibers at stage 6 of WT (A), Dys ${ }^{E 17 \mid E x e l 6184}$ mutant (B), fat2-DeltalCD mutant $(C)$ and Dys, fat2-DeltalCD double mutant (D). Genotype color code is conserved on all panels. $(\mathrm{E}-\mathrm{H})$ Schemes explaining the different parameters that are analyzed in I-L: (E,I) Tissue OP ( $n>7$ follicles for each stage and each genotype); $(F, J)$ Cell OP; $(G, K)$ Local OP; $(H, L)$ difference between Local and Tissue OPs ( $n>171$ cells for each stage and each genotype). (I-L) A two-way ANOVA was performed, followed by applicable post-hoc pairwise comparisons (Tukey). $P$-values between the different genotypes for I,J,K are indicated in Table S3. (M-P) Representative images of wing veins of WT (M), Dys ${ }^{E 17 / E x e / 6184}$ mutant (N), fat2-DeltalCD mutant (O) and Dys, fat2-DeltalCD double mutant (P). ac, anterior crossvein; pc, posterior crossvein. (Q) Quantification of egg elongation for the indicated genotypes ( $n>20$ eggs for each genotype, unpaired $t$-test). (R) Quantification of wing defects observed in the different genotypes on the pc. 'Non analyzable' corresponds to wrinkled or misfolded wings for which crossveins cannot be observed ( $n=40$ wings). For all panels, error bars represent s.d.; ${ }^{* * *} P<0.0001$. Scale bars: $10 \mu \mathrm{m}$. 
These additive effects revealed by these experiments indicate that the DAPC and fat 2 act partially redundantly to orient stress fibers during early stages. They further suggest that Fat 2 and the DAPC are part of a same functional network during follicle elongation, although they probably work in parallel once BM fibrils are deposited.

Intriguingly, Dys, $D g$ and fat 2 mutants show a very specific defect in the wing, with a partial absence of the posterior crossvein, although the cellular basis for this defect is unknown (Christoforou et al., 2008; Viktorinová et al., 2009) (Fig. 4M,N,R). However, this defect is not observed in a fat2/fat2-DeltaICD hypomorphic background (Fig. 4O,R). In the double-mutant Dys, fat2-DeltaICD flies, the posterior crossvein is usually completely absent or limited to a dot, and the anterior crossvein, which is never affected in Dys mutant flies, is incomplete (Fig. 4P,R). These defects indicate a strong genetic interaction in the wings, as observed in follicles.
Thus, these results tend to confirm that Fat2 and the DAPC work in the same network, which might be involved in many contexts.

\section{The DAPC is required for stress fiber orientation during late stages}

Elongation curves of Dys and $D g$ mutants indicate that the major elongation defect appears after stage 12 (Fig. 1H). Of note, for unknown reasons, stress fibers lose their planar polarization at stage $10 \mathrm{~B} / 11$ but regain their proper orientation around stage 12 (Delon and Brown, 2009; Wahlström et al., 2006) (Fig. 5A,B). In WT follicles, this transition can be observed by the change in angular distribution between stage 11 and stage 13 (Fig. 5C,D). F-actin staining of Dys and $D g$ null mutant follicles indicates that the stress fibers lose their orientation at stage 11 as in WT, but fail to regain their proper orientation at stage 13 (Fig. 5E,F,I,J). Angular distribution quantification even indicates a tendency of the stress fibers to be
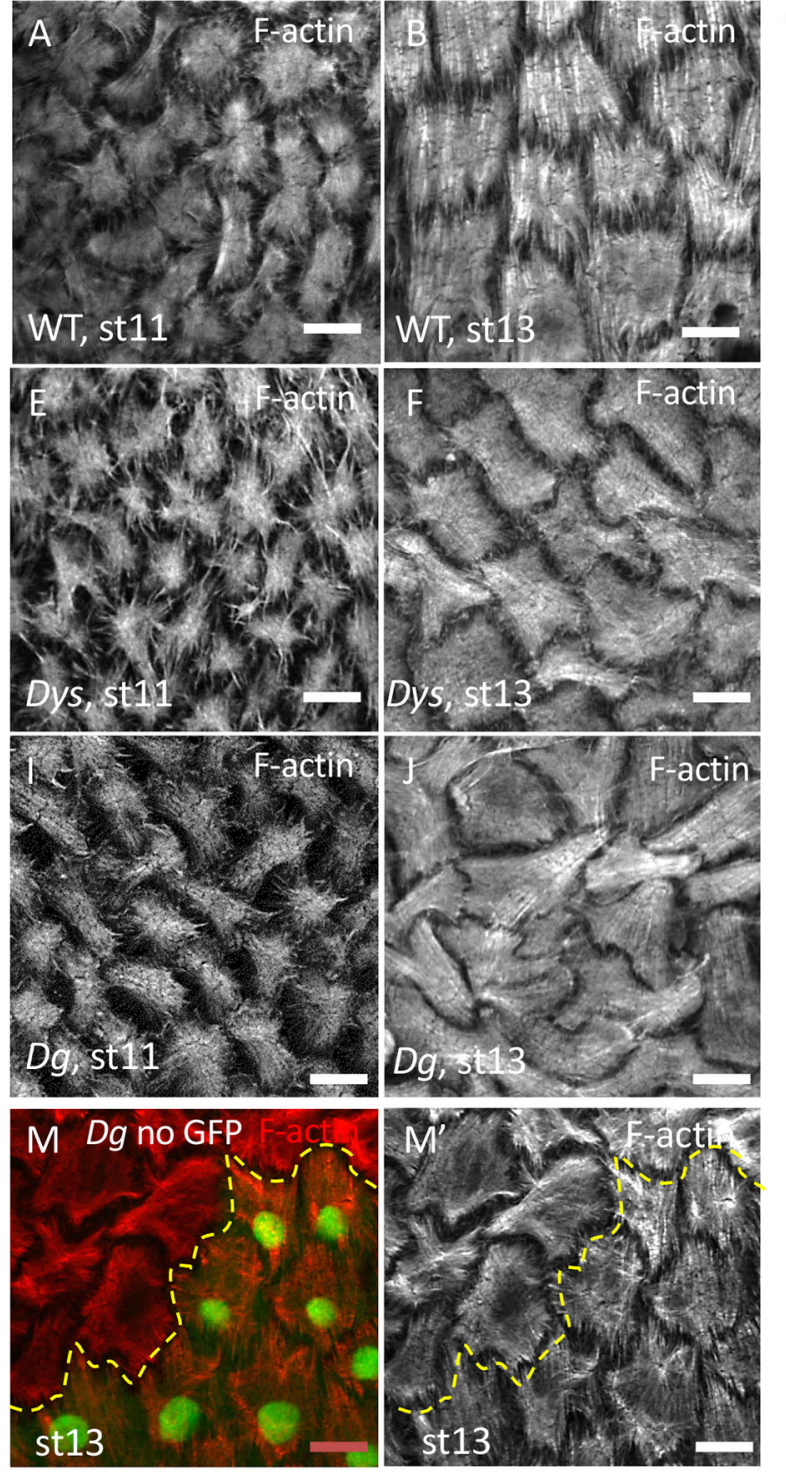
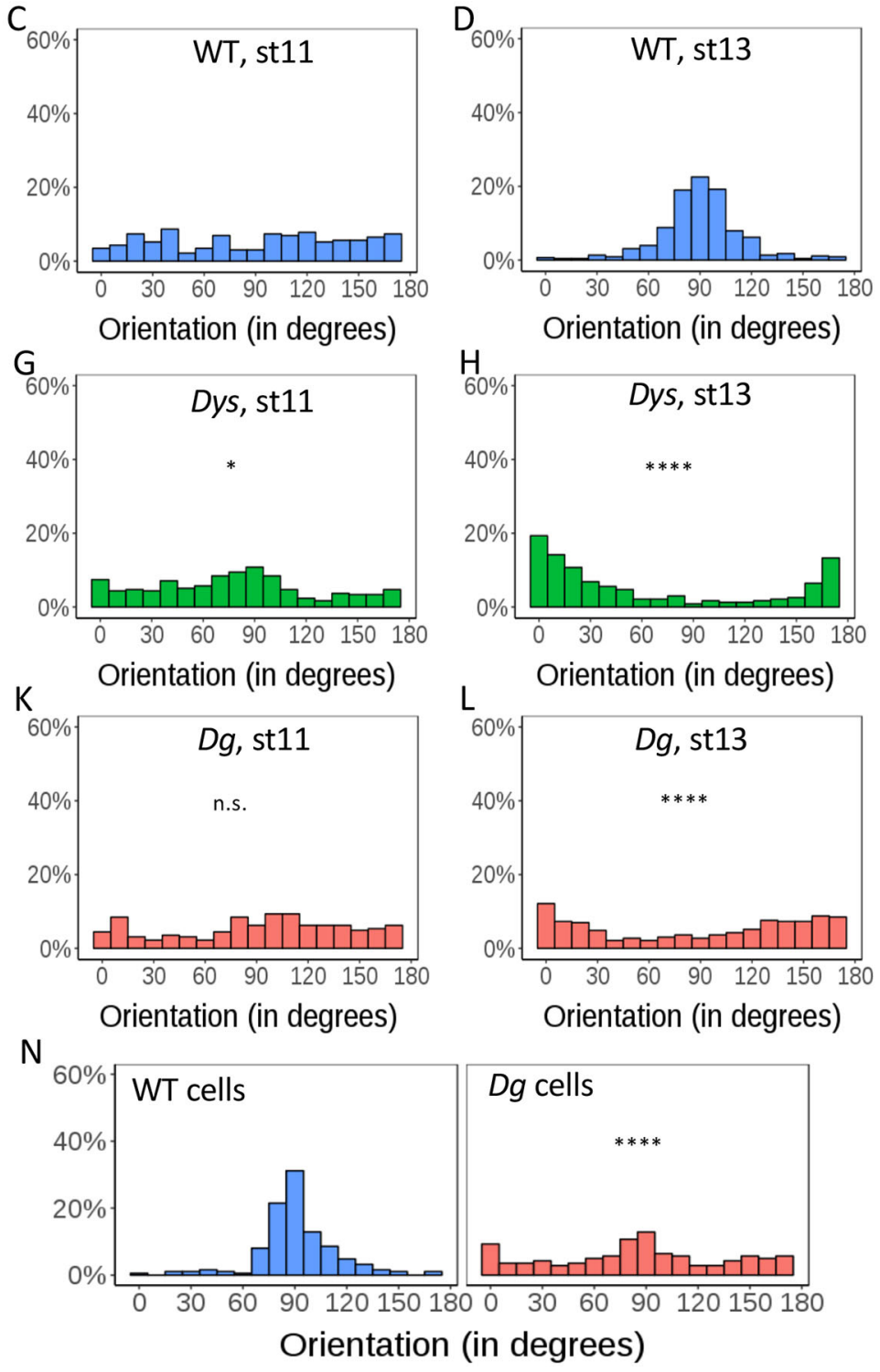

Fig. 5. Stress fiber orientation defect in DAPC mutants during late stages. (A-L) Representative images of basal F-actin in WT (A,B), Dys ${ }^{E 17 / E x e 6184}$ (E,F) and $D g^{\mathrm{O} 86 / \mathrm{O} 43}(\mathrm{I}, \mathrm{J})$ follicles at stages 11 and 13 and quantification of the corresponding angular distribution $(\mathrm{C}, \mathrm{D}, \mathrm{G}, \mathrm{H}, \mathrm{K}, \mathrm{L})(n>5$ follicles). (M-N) Representative image of basal F-actin in $D g$ mutant clone marked by the absence of GFP $\left(\mathrm{M}, \mathrm{M}^{\prime}\right)$ and quantification of the corresponding angular distribution of stress fibers in the mutant cells and the neighboring WT cells $(\mathrm{N})(n>5$ clones ). Angular distribution was compared with that of WT at same stage (and statistically analyzed as described in the Materials and Methods); ${ }^{*} P<0.01,{ }^{* * *} P<0.0001$. n.s., not significant. Scale bars: $10 \mu \mathrm{m}$. 
aligned parallel to the AP axis at stage 13 (Fig. 5G,H,K,L). Despite their misorientation, these fibers appear normal in structure with, for instance, a correct localization of integrins at their extremities (Fig. S3A-C). Importantly, mitotic recombination to generate mosaic tissues indicates that this function is cell-autonomous for $D g$ with misaligned stress fibers only in the mutant cells (Fig. 5M-N), as previously observed in Dys clones in early stages. The defect is strictly cell-autonomous as it is also observed in mutant cells that are adjacent to WT cells, whereas WT cells that are adjacent to mutant cells have stress fibers properly oriented (Fig. 5M). Importantly, this cannot be a consequence of a primary effect on BM fibril deposition because follicle rotation has moved the position of the clone with respect to the BM. It reveals, therefore, another function of the DAPC during follicle development: given the known link of the DAPC with F-actin, it suggests a direct role in stress fiber orientation, sensing and transmitting a cue defining this planar polarization.

\section{Late DAPC function relies on both early and late activities}

Because Dys and Dg mutants affect BM fibril deposition and stress fiber orientation, we aimed to determine which of these defects is the main contributor to the follicle elongation phenotype. To address this question, we took advantage of the temporality of the DAPC function during oogenesis, being required for BM fibril deposition during early stages and for stress fiber orientation mainly during later stages. Importantly, these three defects - follicle elongation, BM fibril deposition and stress fiber orientation - are recapitulated when RNAi against Dg is induced in follicle cells during all stages using a traffic jam:Gal4 ( $t j: \mathrm{Gal} 4)$ driver, expressed in follicle cells throughout oogenesis (Fig. 6F-I). We also used Cy2: Gal4, which is only expressed in follicle cells from stage 9 (Fig. $\mathrm{S} 4 \mathrm{~N})$. The expression of $D g$ RNAi with this driver knocks it down after the correct BM fibril deposition (Fig. S4A,B), but before the second phase of stress fiber orientation. As expected, the stress fiber orientation is disrupted at stage 13, confirming that this DAPC function is distinct from the one influencing the BM (Fig. S4C,D). Importantly, egg elongation is also affected (Fig. S4E). Thus, BM fibrils are not sufficient to drive late follicle elongation, and stress fiber orientation is necessary for it.

We also performed the reverse experiment by trying to rescue the late stress fiber orientation defect in a $D g$ mutant using a UAS:Dg transgene. Expression of Dg during the whole oogenesis in the follicle cells, using the $t j:$ Gal4 driver in a mutant background, rescues the elongation defect, the BM fibril deposition and stress fiber orientation (Fig. S4F,H,I,J,M). We then tried to rescue the $D g$ mutant using the Cy2:Gal4 driver, which clearly allows Dg detection from stage 9 with an antibody failing to detect endogenous level of the protein (Fig. S4N-P'). As expected, such follicles show BM defects associated with $D g$ mutation (Fig. S6G,H). However, surprisingly, this genetic combination also fails to rescue stress fiber orientation (Fig. S4K,L). Moreover, egg elongation is only weakly rescued (Fig. S4M). Together, these results indicate that late expression of $\mathrm{Dg}$ is not sufficient to restore its late functions, and, thus, that these late functions also rely on a previous role of $\mathrm{Dg}$ during oogenesis.

To confirm these important observations, we set up an alternative method to control the timing of Dg inhibition by RNAi, using $t j$ : Gal4 and Tub:Gal80 ${ }^{\text {ts }}$, which is a thermosensitive Gal4 inhibitor allowing a temporal control of its activity. We defined two conditions allowing either Gal4 activity during early stages (switch from 30 to $18^{\circ} \mathrm{C}$, 'Early $D g$ RNAi') or late stages (switch from 18 to $30^{\circ} \mathrm{C}$, 'Late $D g$ RNAi') (Fig. 6A). Moreover, positive and negative controls were performed at $18^{\circ} \mathrm{C}$ and $30^{\circ} \mathrm{C}$ (Fig. 6). For each condition, an internal negative control (driver line) with the same temperature was performed. $D g$ RNAi at $18^{\circ} \mathrm{C}$ is similar to control (Fig. 6B-E) whereas at $30^{\circ} \mathrm{C}$ it recapitulates all the defects observed in $D g$ mutants, as previously mentioned (Fig. 6F-I). The best timing for the temperature switches was adjusted by checking their effect on fibril in late stages, taking into account that these fibrils are formed during early stages. Thus, fibrils are normal in $18-30^{\circ} \mathrm{C}$ conditions and affected in $30-18^{\circ} \mathrm{C}$ conditions (Fig. $6 \mathrm{~J}, \mathrm{~N}$ ). We observed that F-actin orientation at late stages and follicle elongation are affected in both late (Fig. 6K-M) and early (Fig. 6OQ) $D g$ RNAi conditions, confirming our previous results. Thus, the DAPC is required at two different time windows and its second function is dependent on the first one.

\section{BM fibril deposition provides a persistent cue for F-actin orientation}

Overexpression of Rab10 increases the BM fibril fraction and enhances follicle elongation, a phenotype opposite to the loss of function of the DAPC (Isabella and Horne-Badovinac, 2016). We therefore performed an epistasis test between these two conditions. Concomitant overexpression of Rab10 and knockdown of Dys in follicle cells using $t j$ :Gal4 increases the amount of BM fibrils to a higher level than that of the WT control and thus fully compensates for the absence of Dys (Fig. S5A-D,E). This rescue is not due to titration of the Gal4 protein by the second UAS line when we used both Dys RNAi and UAS:Rab10 constructs as the BM fibril fraction in a UAS: tomato; Dys RNAi control is not increased (Fig. S5I). Classical interpretation of such a test would indicate that Rab10 acts downstream (or in parallel) of the DAPC. These experiments were performed at optimal temperature for Gal4-induced expression $\left(30^{\circ} \mathrm{C}\right)$. However, in this context Rab10 overexpression disrupts follicle elongation (Isabella and Horne-Badovinac, 2016), and it is also associated with the abnormal targeting of ColIV-containing vesicles to the apical side of the cells (Fig. S5H). Therefore, these conditions did not allow us to determine whether it could also rescue follicle elongation. As a consequence, we repeated these experiments at $25^{\circ} \mathrm{C}$ and observed an almost complete rescue of $\mathrm{BM}$ fibril fraction (Fig. 7A). However, mature egg elongation was still disrupted, as in DAPC loss of function (Fig. 7B). Thus, for elongation, Dys should be placed downstream of Rab10. Taken together, these results are consistent with the proposal that the DAPC has two separate functions, one upstream and one downstream of Rab10. Moreover, it also confirms that, in absence of the DAPC, BM fibrils are not sufficient for elongation.

We also analyzed stress fiber orientation in late stages in this context. Angular distribution for Dys RNAi (Fig. S5J) compared with that of null mutants (Fig. $5 \mathrm{H}$ ), indicates that it corresponds to hypomorphic conditions. This result prompted us to also analyze the different order parameters, as previously done at early stages, to get a more precise evaluation of stress fiber orientation. The three parameters are strongly affected in Dys RNAi (Fig. 7C-E). When Rab10 is overexpressed at the same time we observed a partial rescue of this defect on the angular distribution, the Tissue OP and the Local OP but not the Cell OP (Fig. S5J, Fig. 7C-E,I). These observations could be explained by the rescue of the early function of the DAPC by Rab10 and the hypomorphic nature of Dys RNAi. Nonetheless, this approach offers a unique opportunity to test whether the DAPC's first function in BM fibril deposition is required for its later function in stress fiber orientation. We repeated the experiments but allowing Dys expression during late stages by a temperature switch $\left(25^{\circ} \mathrm{C}\right.$ to $\left.18^{\circ} \mathrm{C}\right)$. Strikingly, the late expression of Dys promotes correct stress fiber orientation on all the parameters 


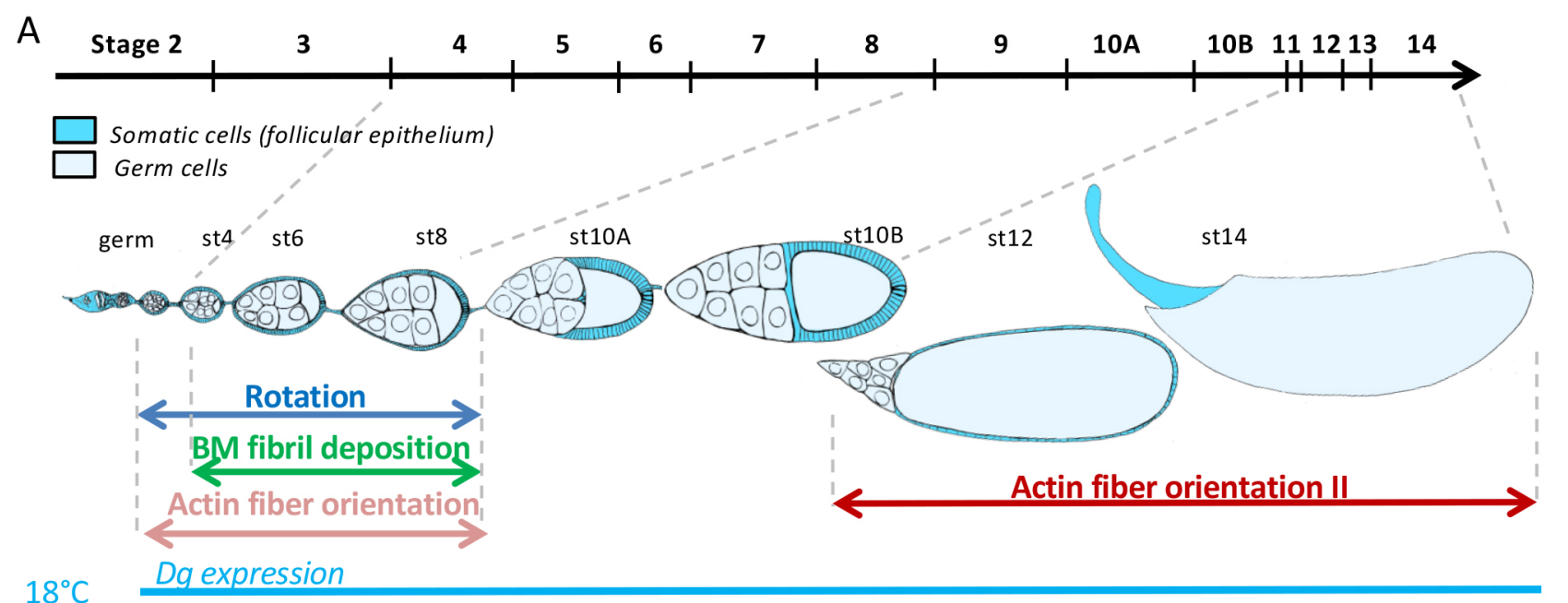

$30^{\circ} \mathrm{C}$

$18-30^{\circ} \mathrm{C}$

$30-18^{\circ} \mathrm{C}$
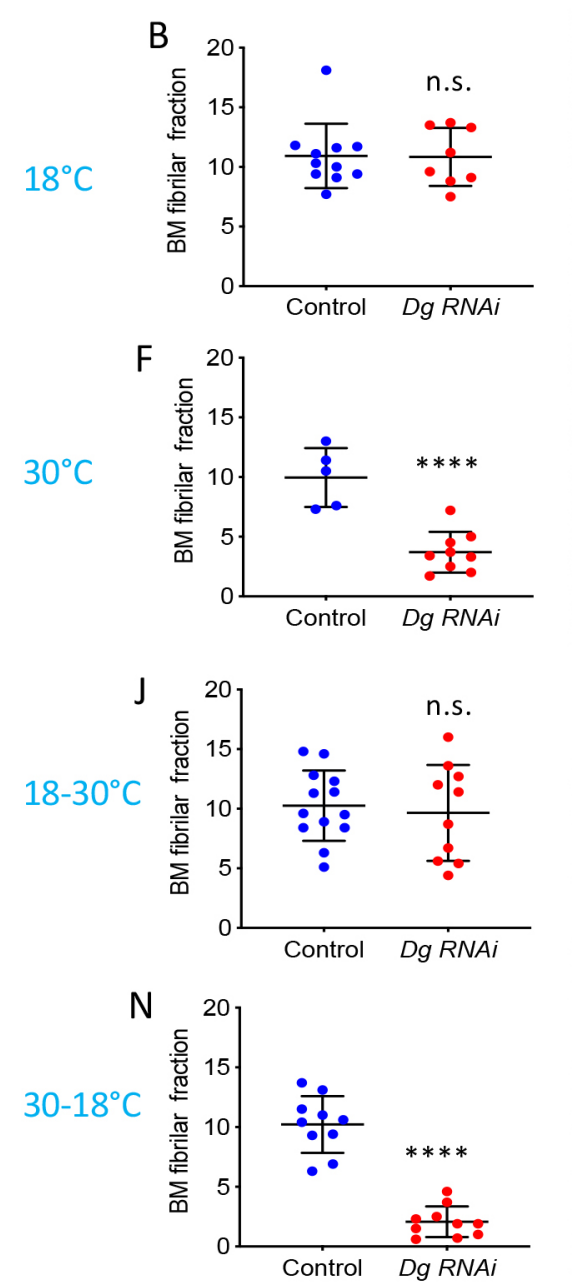
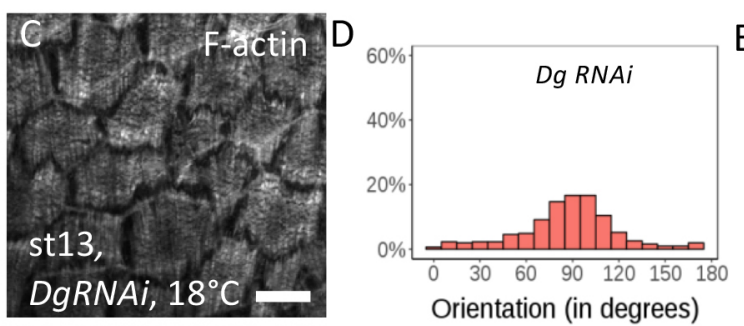

$\mathrm{E}$
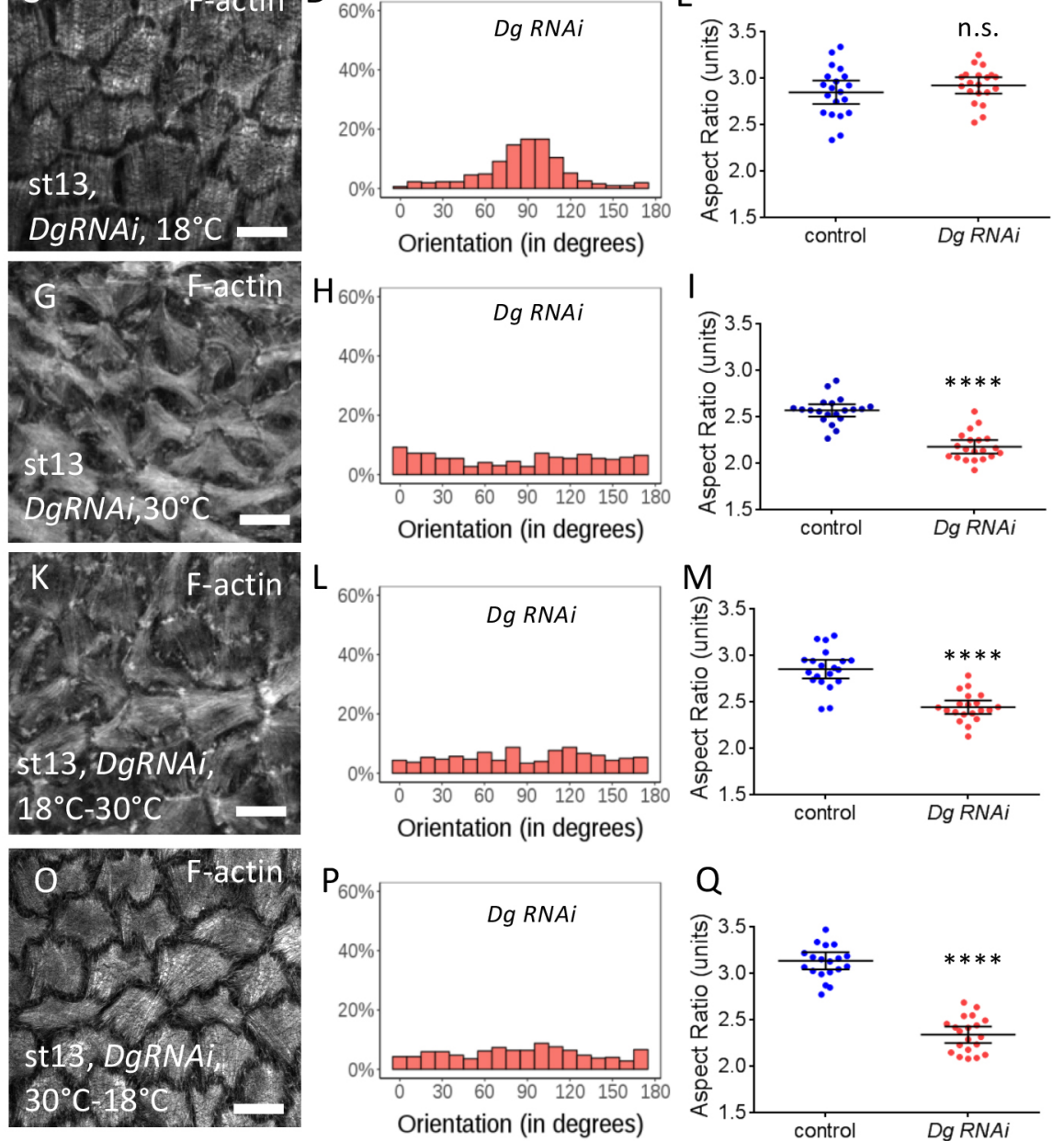

Fig. 6. The DAPC is required at two time frames for follicle elongation. (A) Scheme of an ovariole with the main events involved in follicle elongation. The top line indicates the time scale of the different developmental stages. The ovariole is oriented anterior to posterior (germ, germarium). The level of endogenous $\mathrm{Dg}$ is temporally controlled by the temperature (blue lines). (B,F,J,N) Quantification of the BM fibril fraction of $D g$ RNAi stage 12 follicles expressing CollV-GFP in the indicated condition compared with control performed in the same temperature condition ( $n>5$ follicles). (C,D,G,H,K,L,O,P) Representative images of basal F-actin of $D g$ RNAi stage 13 follicles in the indicated condition $(\mathrm{C}, \mathrm{G}, \mathrm{K}, \mathrm{O})$ and quantification of the corresponding angular distribution (D,H,L,P) ( $n>5$ follicles). (E,I,M,Q) Aspect-ratio quantification of temporally controlled Dg RNAi mature compared with control performed in the same temperature condition ( $n>20$ eggs). For all panels, error bars represent s.d.; ${ }^{* * * *} P<0.0001$ (unpaired $t$-test). n.s., not significant. Scale bars: $10 \mu \mathrm{m}$. 


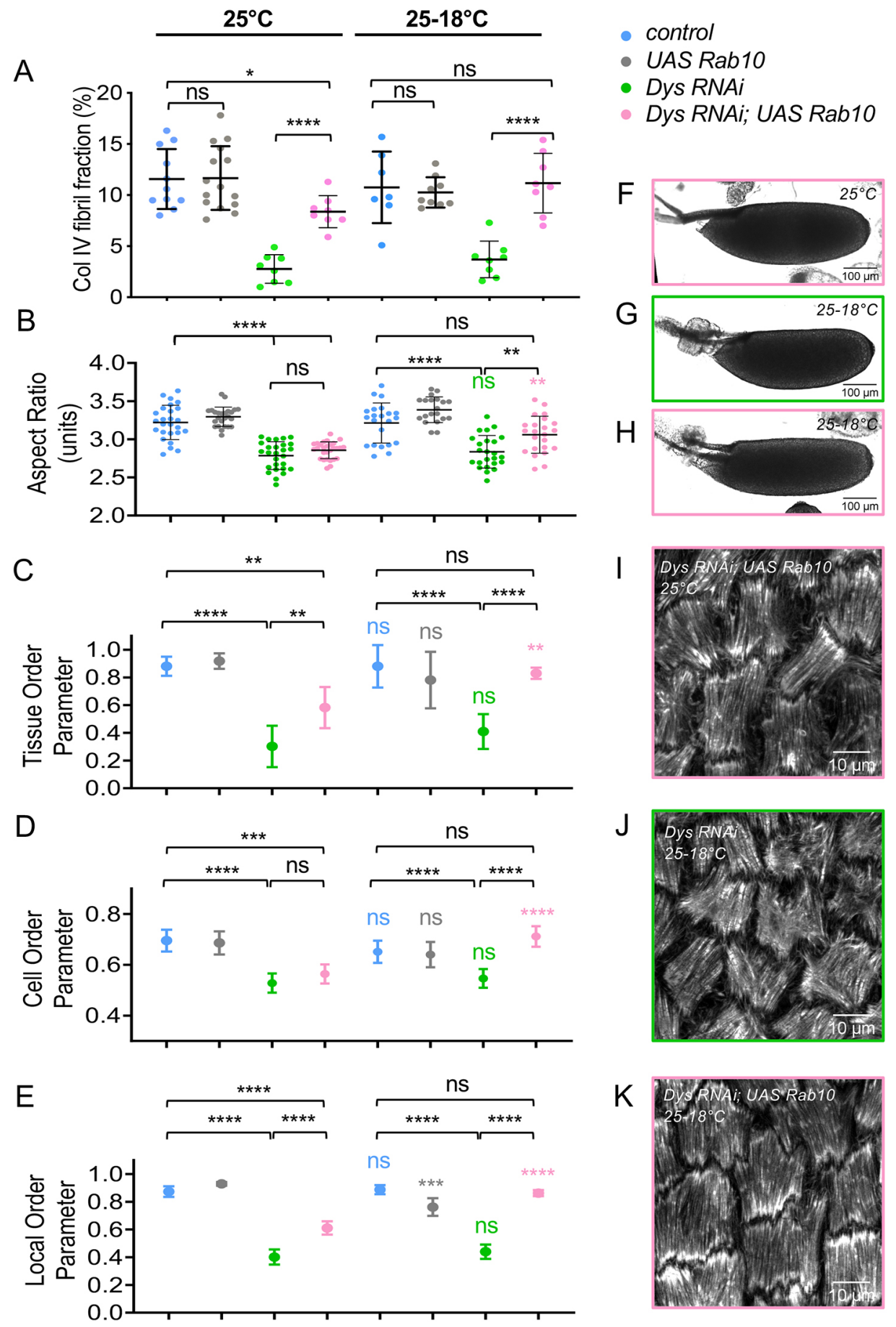

Fig. 7. BM fibrils provide a cue for DAPCdependent $\mathrm{F}$-actin orientation.

(A) Quantification of the BM fibril fraction at stage 8 for the indicated genotypes at $25^{\circ} \mathrm{C}(n>8)$. (B-E) For the indicated genotypes, without $\left(25^{\circ} \mathrm{C}\right)$ or with $\left(25-18^{\circ} \mathrm{C}\right)$ a switch to nonpermissive temperature, quantification of the aspect-ratio of mature eggs (B), the Tissue-OP ( $n>7$ follicles) (C), the Cell-OP (D), and the Local-OP at stage 13 ( $n>45$ cells) (E) $(\mathrm{A}, \mathrm{B})$ unpaired $t$-test; (C-E) two-way ANOVA performed, followed by applicable post-hoc pairwise comparisons (Tukey). (F-K) Representative images of mature eggs $(\mathrm{F}-\mathrm{H})$ and of stress fibers (I-K) at stage 13 for the indicated genotypes without $\left(25^{\circ} \mathrm{C}\right)$ or with $\left(25-18^{\circ} \mathrm{C}\right)$ a switch to non-permissive temperature. For all panels, error bars represent s.d.; ${ }^{*} P<0.01,{ }^{* *} P<0.005,{ }^{* * *} P<0.001$, ${ }^{* * * *} P<0.0001$. Colored asterisks (or ns) correspond to the statistical test for the same genotype between $25^{\circ} \mathrm{C}$ and $25-18^{\circ} \mathrm{C}$ conditions. ns, not significant. that we analyzed only if the BM fibrils have been restored by Rab10 overexpression (Fig. 7C-E,J,K, Fig. S5J). Moreover, this condition also restores egg elongation (Fig. 7B,F-H). These experiments demonstrate the double function of the DAPC, with the latter in $\mathrm{F}$-actin organization depending on the first one in BM structure. It also shows that the BM fibrils are required for F-actin orientation long after their deposition and that this function explains their contribution to follicle elongation.

\section{DISCUSSION}

In this article, we show that the DAPC contributes to follicle elongation by two distinct functions in which the second relies on the output of the first one. These results are important for understanding the activity of this key complex and the morphogenetic mechanisms involving the ECM.

\section{Follicle elongation relies on multiple mechanisms}

Genetic data has already demonstrated that follicle elongation relies on at least two different and successive mechanisms. The first is controlled by JAK-STAT and involves the follicle cell apical domain, whereas the second is controlled by Fat 2 and involves the basal domain and the BM (Alégot et al., 2018; Aurich and Dahmann, 2016). Between these phases, around stage 7-8, JAKSTAT and Fat2 seem to be integrated in a third mechanism based on a BM stiffness gradient (Crest et al., 2017). Interestingly, a very recent report suggests that this gradient may not directly influence 
tissue shape but rather do so by modifying the properties of the follicle cells underneath (Chen et al., 2019). Here, we show that the DAPC influences elongation mainly at very late stages, suggesting the existence of a fourth mechanistic elongation phase. Of note, elongation at these late stages is also defective in fat 2 mutants (Alégot et al., 2018; Aurich and Dahmann, 2016). This is consistent with the fact that rotation is required for polarized BM fibril deposition, and that this deposition depends on and is required for DAPC function. The existence of multiple and interconnected mechanisms to induce elongation, a process that initially appeared to be very simple, highlights the true complexity of morphogenesis, and the necessity to explore it in simple models.

\section{BM fibrils act as a planar polarity cue and F-actin as a molecular corset}

Fat2 is clearly part of the upstream signal governing the basal planar polarization. However, how this polarization leads to tissue elongation is still debated. It has been proposed that elongation relies on a molecular corset that could be formed, non-exclusively, by BM fibrils or F-actin stress fibers (Gutzeit, 1990; Gutzeit et al., 1991; Haigo and Bilder, 2011; Frydman and Spradling, 2001; Bateman et al., 2001; Viktorinová et al., 2009; Cetera et al., 2014). The initial observation that rotation is required for both elongation and BM fibrils favored a direct mechanical role for these structures (Haigo and Bilder, 2011). Recent data showing that BM fibrils are stiffer than the surrounding ECM supports this view (Chlasta et al., 2017). Moreover, increasing the BM fibril number and size can lead to hyper-elongation (Isabella and Horne-Badovinac, 2016). Finally, addition of collagenase induces follicle rounding, at least at some stages, and genetic manipulation of the ECM protein levels also influences elongation (Haigo and Bilder, 2011; Isabella and Horne-Badovinac, 2015a,b; Chlasta et al., 2017). However, these experiments did not discriminate between the function of the fibril fraction and a general BM effect. Moreover, they do not demonstrate whether their impact on elongation is direct and mechanic or, indirect by a specific response of the epithelial cells. Fat2 and rotation are also required for the proper orientation of the stress fibers. The F-actin molecular corset is dynamic with follicle cells undergoing basal pulsations, and perturbation of both these oscillations and of the stress fiber structure affect elongation (Bateman et al., 2001; He et al., 2010). In the DAPC mutants, we observed a faint but significant elongation defect during mid-oogenesis and a stronger one after stage 12 . These defects are clearly correlated with the stress fiber orientation defects observed in the same mutants, both temporally and in terms of intensity. Moreover, although overexpression of Rab10 in a Dys lossof-function mutant restores BM fibrils, it does not rescue elongation, indicating that stress fiber orientation is instrumental.

Thus, if the role of the BM fibrils as a direct mechanical corset appears limited, what is their function? One possibility could have been that they promote rotation, acting by positive feedback and explaining the speed increase over time. However, the rotation reaches the same speed in WT and DAPC mutants, excluding this possibility. Similarly, increasing the fibril fraction also has no effect on rotation speed (Isabella and Horne-Badovinac, 2016).

Our results strongly argue that BM fibrils act as a cue for the orientation of stress fibers, which then generate the mechanical strain for elongation. This appears clear in late stages when the function of the DAPC for stress fiber orientation is dependent on the previous BM fibril deposition. Although it is unknown why the cells lose their orientation from stages 10 to 12 , the BM fibrils provide the long-term memory of the initial PCP of the tissue, allowing stress fiber reorientation (Fig. 8). Such a mechanism appears to be a very efficient way to memorize positional cues, and could represent a general BM function in many developmental processes.

How BM fibrils contribute to stress fiber orientation remains to be elucidated. Nevertheless, we can hypothesize that it involves their higher stiffness or a higher density in binding sites for ECM receptors. Our data indicate that the DAPC might be such a receptor. This hypothesis is supported by our findings that the DAPC acts in a cell-autonomous manner and promotes stress fiber orientation in each cell and acts locally rather than at a tissue scale. Of note, this kind of PCP is unusual because it does not require signal transmission between neighboring cells, usually through heterophilic protein-protein interactions. However, an interesting parallel with the PCP established by Fat/Dachsous and the core PCP pathways could be drawn. In many tissues, these two pathways work in parallel and their cooperation is required for proper PCP establishment at the apical domain of epithelial cells (Hale and Strutt, 2015). The genetic interaction that we revealed between Fat2 and the DAPC suggests that robust basal PCP establishment also requires the integration of two independent mechanisms. Importantly, this genetic interaction applies to at least two tissues, suggesting a general functional link between them.

\section{The DAPC acts as an organizer of the basal domain of epithelial cells}

We found that the DAPC impacts the two key actors at the basal domain of the follicle cells: the BM and the stress fibers linked to the

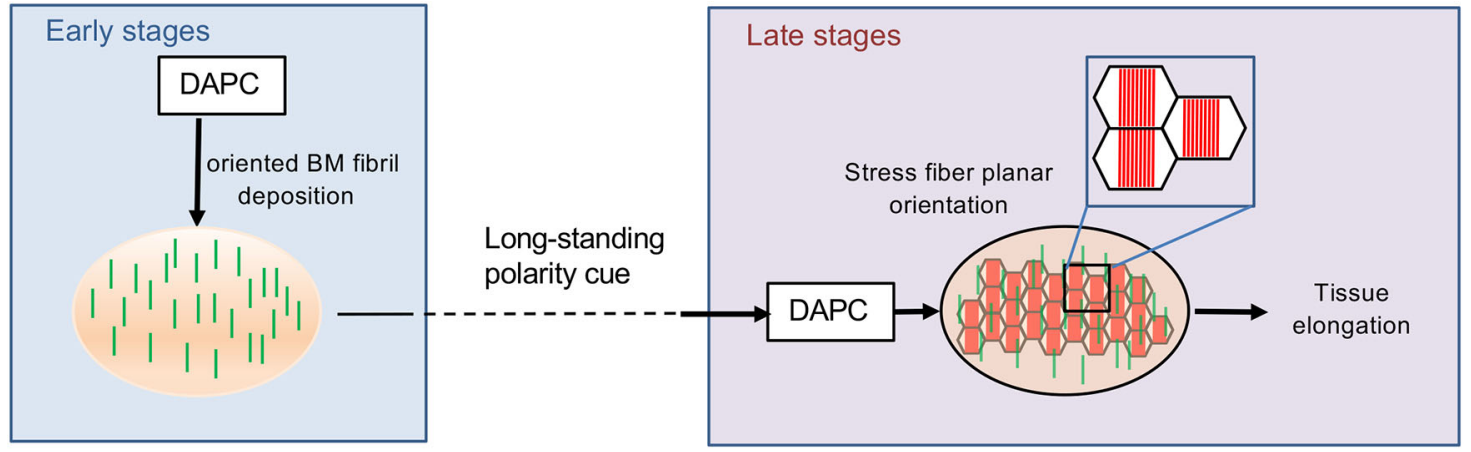

Fig. 8. Dual function of the DAPC during elongation revealing that BM fibrils work as a persistent planar polarity cue. During the early stages (4-8) the DAPC participates in the formation of oriented BM fibrils perpendicular to the follicle elongation axis. These fibrils are maintained during follicle development including stages with intense morphogenetic processes unrelated to elongation (stages 9-11). At late stages, cells use the persistent BM fibrils as a signal to guide the planar orientation of the stress fibers. This event depends on a distinct second function of the DAPC. The stress fibers are the main mechanical effector of follicle elongation. 
BM. All the defects of $D g$ null mutants were also observed in Dys mutants, demonstrating a developmental and morphogenetic role for this gene. In vertebrates, at least in some tissues, Dg presence appears to be essential for BM assembly (Henry and Campbell, 1998; Satz et al., 2010; Bello et al., 2008; Buisson et al., 2014). However, BM formation on the follicular epithelium does not require $\mathrm{Dg}$, suggesting the existence of alternative platforms for its general assembly. Our genetic data suggest that Rab10 is epistatic to Dg for BM fibril deposition. The usual interpretation of such a result would be that Dg is involved in the targeting of ECM secretion upstream of Rab10 rather than in ECM assembly in the extracellular space. In Caenorhabditis elegans, Dg acts as a diffusion barrier to define a precise subcellular domain for ECM remodeling (Naegeli et al., 2017). One could imagine that the DAPC has a similar function in follicle cells, by defining the position where the Rab10 secretory route is targeted. However, in DAPC loss of function, some ECM is still secreted between cells, suggesting that the lateral Rab10 route is not affected. Moreover, ECM proteins do not abnormally accumulate between cells in such mutants, suggesting that they are able to leave this localization but without forming BM fibrils. Therefore, the functional interplay between Rab10 and the DAPC is still unclear.

As mentioned before, $\mathrm{Dg}$ has often been proposed to act as a scaffold to promote BM assembly in mice. Deletion of the Dg intracellular domain is only sub-lethal in mice, whereas complete loss of this protein is lethal very early during development, indicating that the abolishment of Dg's interaction with Dys affects its function only partially (Henry and Campbell, 1998; Satz et al., 2009). In these mice, laminin assembly can still be observed, for instance in the brain and retina (Satz et al., 2010; Clements et al., 2017). Similar results were also obtained in cultured mammary epithelial cells (Weir et al., 2006). Thus, despite the existence of Dys paralogs that could mask some effects on ECM and the fact that we observed the same ECM alteration in $D g$ or Dys mutant fly follicles, not all the Dg functions related to ECM assembly or secretion involve Dys. It is possible that Dys is required when Dg needs a very specific subcellular targeting for its function, whereas a more general role in ECM assembly would be independent of Dys. Our results suggest that some specific effects of Dys on ECM could have been underestimated and this could help to explain the impact of its loss of function on tissue integrity maintenance. For instance, as it has been reported that Dg influences ECM organization in fly embryonic muscles, it would be interesting to determine whether this also involves Dys (Yatsenko and Shcherbata, 2014).

The DAPC is involved in planar polarization of the basal stress fibers and its ability to read ECM structure to orchestrate integrindependent adhesion could play a role in many developmental and physiological contexts. The link between the ECM and F-actin provided by this complex is likely required for this function, although this remains to be formally demonstrated. BM fibrils could provide local and oriented higher density of binding sites for $\mathrm{Dg}$, and the alignment could then be transmitted to the actin cytoskeleton. Alternatively, DAPC function could rely on sensing the mechanical ECM properties. The hypothesis that the DAPC could act as a mechanosensor is a long-standing proposal, partly due to the presence of spectrin repeats in Dys (Brown and Lucy, 1993; Garbincius and Michele, 2015). The basal domain of the follicle cells may offer an amenable model to combine genetics and cell biology approaches to decipher such function.

Altogether, this work provides important insights on the role of the BM during morphogenesis, by acting as a static PCP cue retaining spatial information while cells are highly dynamic. It also reveals important functions of the DAPC, including Dys, that may be broadly involved during animal development and physiology.

\section{MATERIALS AND METHODS \\ Genetics}

The detailed genotypes, temperature and heat-shock conditions are given in Table S1.

\section{Live imaging and rotation speed analysis}

For live imaging, ovaries were dissected, as described previously (Alégot et al., 2018), and stained with the membrane dye FM4-64. Samples were cultured for less than $1 \mathrm{~h}$ before imaging. Damaged follicles (identified on the basis of abnormal FM4-64 incorporation) were not analyzed. Rotation speed analysis was performed using a Fiji Manual Tracking plug-in. For each follicle, speed was measured at three independent positions during $30 \mathrm{~min}$.

\section{Dissection and immunostaining}

Resources and reagents are listed in Table S2. Dissection and immunostaining were performed as described previously (Vachias et al., 2014 ) with the following changes: ovaries were dissected in supplemented Schneider medium, and ovarioles were separated and the muscle sheath was removed before fixation to obtain undistorted follicles. Stage was determined using unambiguous reference criteria that are independent of follicle shape (Spradling et al., 1993). For quantification of stress fiber orientation, ovaries were fixed with $8 \%$ paraformaldehyde (PFA) containing phalloidin-TRITC or phalloidin Atto-488. They were then permeabilized with $0.02 \%$ Triton X-100 in PBS at $0.02 \%$ and incubated again with phalloidin-TRITC (or Atto-488). Images were taken using a Leica SP8 or a Zeiss LSM800 Airyscan confocal microscope except for stage 12 BM images, which were acquired on an inverted Zeiss Spinning Disc (Yokogawa CSU-22). Immunostaining of pericellular ECM was performed as described by Isabella and Horne-Badovinac (2016), using antibodies against GFP (ColIV-GFP-expressing flies) or against LanA (a gift from T. Volk, Weizmann Institute, Rehovot, Israel).

\section{Aspect ratio determination}

The length of the long and short axes of each follicle were directly measured by transmitted light microscopy and used to determine the aspect ratio.

\section{BM fraction, pericellular matrix and F-actin orientation quantification}

BM fibril fraction was determined using a homemade Fiji macro, using the principles developed by Isabella and Horne-Badovinac (2016). Threshold for fibril fraction determination was manually adjusted on each control experiment to obtain accurate fibril detection, taking into account the slight variation of the fluorescence depending on the temperature, the developmental stage and the microscope used. The same threshold was then assigned for all the genotypes of the same experiment. The pericellular matrix was quantified using the Fiji tool to measure the 'meangray value' of a $1.32-\mu \mathrm{m}$-wide line drawn on the cell walls (about 100 lines for each genotype, 20 cells).

Stress fiber orientation and Tissue OP analysis were performed as described by Cetera et al. (2014), after manual segmentation. Analyses were performed on 40 and $96 \mu \mathrm{m}^{2}$ images for the early and late stages, respectively. The Cell OP was determined in the same way, by analyzing the alignment robustness at the cellular scale. For Local OP, the average cell radius of each image was determined. Then, we analyzed the cells with their barycenter being in 3.5 radiuses from the considered cell, this value fitting with an average of six neighbors. Clusters with fewer than four cells were excluded. Then, the radius was progressively increased from 3.5 to 25 . At 25 , all the cells of the image were included, and thus the Local OP value corresponded to the Tissue OP value. Therefore, this approach was used to observe the tendency from Local OP to Tissue OP and to calculate for each cell the difference between them. As the orientation of the fibers is only between $0^{\circ}$ and $180^{\circ}$, the OP, as it has been defined, is a measure of the variability among corresponding mean 
directors, independently of the scale at which it is used: local directors were used for the Cell OP whereas the mean cell orientation was used to compute the OP for larger scales.

\section{Statistical analyses}

For all experiments, the minimal sample size is indicated in the figure legends. Results were obtained from at least two independent experiments, and for each experiment multiple females were dissected. Randomization or blinding was not performed. The normality of the samples was calculated using the D'Agostino and Pearson normality test. The unpaired $t$-test was used to compare samples with normal distribution, and the unpaired MannWhitney test for samples without normal distribution.

Concerning the stress fiber angular distribution, the circular mean orientation of the fibers was computed for each cell and then used for the Rao's test of homogeneity. For each stage, a pairwise comparison of genotypes was performed to determine whether the polar vectors or the dispersions were equal. These tests were performed in R using the 'circular' package.

\section{Acknowledgements}

We thank R. Ray, H. Ruohola-Baker and T. Volk for fly stocks or reagents. We also thank the CLIC facility (Clermont Imagerie Confocale), and team members for comments on the manuscript.

\section{Competing interests}

The authors declare no competing or financial interests.

\section{Author contributions}

Conceptualization: F.C.C., H.A., C.F., A.I., S.H.-B., V.M.; Methodology: F.C.C., C.D. H.A., O.B., V.M.; Software: P.P.; Validation: F.C.C., C.D., V.M.; Formal analysis: F.C.C., C.D., H.A., P.P., V.M.; Investigation: F.C.C., C.D., H.A., C.F., A.I.; Data curation: H.A.; Writing - original draft: F.C.C., S.H.-B., V.M.; Visualization: F.C.C. C.D., P.P., V.M.; Supervision: S.H.-B., V.M.; Project administration: V.M.; Funding acquisition: S.H.-B., V.M.

\section{Funding}

This work was supported by the Association Française contre les Myopathies (AFM) (Trampoline grant 17683 and MyoNeurAlp network). This research was also financed by the French government (Ministère de l'Enseignement Supérieur et de la Recherche) IDEX-ISITE initiative 16-IDEX-0001 (CAP 20-25) and by a grant from the American Cancer Society (RSG-14-176) to S.H-B. F.C.C. was supported by the Fondation pour la Recherche Médicale (FRM) (FDT20170437189), and A.I. by the National Institutes of Health (T32 HD055164) and a National Science Foundation Graduate Research Fellowship. Deposited in PMC for release after 12 months.

\section{Supplementary information}

Supplementary information available online at

http://dev.biologists.org/lookup/doi/10.1242/dev.186957.supplemental

\section{References}

Alégot, H., Pouchin, P., Bardot, O. and Mirouse, V. (2018). Jak-Stat pathway induces Drosophila follicle elongation by a gradient of apical contractility. Elife 7 , e32943. doi:10.7554/eLife.32943.034

Aurich, F. and Dahmann, C. (2016). A mutation in fat2 uncouples tissue elongation from global tissue rotation. Cell Rep 14, 2503-2510. doi:10.1016/j celrep.2016.02.044

Barlan, K., Cetera, M. and Horne-Badovinac, S. (2017). Fat2 and lar define a basally localized planar signaling system controlling collective cell migration. Dev Cell 40, 467-477.e5. doi:10.1016/j.devcel.2017.02.003

Barresi, R. and Campbell, K. P. (2006). Dystroglycan: from biosynthesis to pathogenesis of human disease. J. Cell Sci. 119, 199-207. doi:10.1242/jcs.02814

Bateman, J., Reddy, R. S., Saito, H. and Van Vactor, D. (2001). The recepto tyrosine phosphatase Dlar and integrins organize actin filaments in the Drosophila follicular epithelium. Curr. Biol. 11, 1317-1327. doi:10.1016/S0960-9822(01) 00420-1

Bello, V., Sirour, C., Moreau, N., Denker, E. and Darribère, T. (2008). A function for dystroglycan in pronephros development in Xenopus laevis. Dev. Biol. 317 106-120. doi:10.1016/j.ydbio.2008.02.024

Brown, S. C. and Lucy, J. A. (1993). Dystrophin as a mechanochemical transducer in skeletal muscle. BioEssays 15, 413-419. doi:10.1002/bies.950150608

Buisson, N., Sirour, C., Moreau, N., Denker, E., Le Bouffant, R., Goullancourt A., Darribère, T. and Bello, V. (2014). An adhesome comprising laminin, dystroglycan and myosin IIA is required during notochord development in Xenopus laevis. Development 141, 4569-4579. doi:10.1242/dev.116103

Cetera, M., Ramirez-San Juan, G. R., Oakes, P. W., Lewellyn, L., Fairchild, M. J., Tanentzapf, G., Gardel, M. L. and Horne-Badovinac, S. (2014). Epithelial rotation promotes the global alignment of contractile actin bundles during Drosophila egg chamber elongation. Nat. Commun. 5, 5511. doi:10.1038/ ncomms6511

Chen, D.-Y., Lipari, K. R., Dehghan, Y., Streichan, S. J. and Bilder, D. (2016) Symmetry breaking in an edgeless epithelium by Fat2-regulated microtubule polarity. Cell Rep 15, 1125-1133. doi:10.1016/j.celrep.2016.04.014

Chen, D.-Y., Crest, J. and Bilder, D. (2017). A cell migration tracking tool supports coupling of tissue rotation to elongation. Cell Rep 21, 559-569. doi:10.1016/j. celrep.2017.09.083

Chen, D.-Y., Crest, J., Streichan, S. J. and Bilder, D. (2019). Extracellular matrix stiffness cues junctional remodeling for 3D tissue elongation. Nat. Commun. 10 3339. doi:10.1038/s41467-019-10874-x

Chlasta, J., Milani, P., Runel, G., Duteyrat, J.-L., Arias, L., Lamiré, L.-A., Boudaoud, A. and Grammont, M. (2017). Variations in basement membrane mechanics are linked to epithelial morphogenesis. Development 144, 4350-4362. doi:10.1242/dev.152652

Christoforou, C. P., Greer, C. E., Challoner, B. R., Charizanos, D. and Ray, R. P. (2008). The detached locus encodes Drosophila Dystrophin, which acts with othe components of the Dystrophin associated protein complex to influence intercellular signalling in developing wing veins. Dev. Biol. 313, 519-532. doi:10. 1016/j.ydbio.2007.09.044

Clements, R., Turk, R., Campbell, K. P. and Wright, K. M. (2017). Dystroglycan maintains inner limiting membrane integrity to coordinate retinal development. J. Neurosci. 37, 8559-8574. doi:10.1523/JNEUROSCI.0946-17.2017

Crest, J., Diz-Muñoz, A., Chen, D. Y., Fletcher, D. A. and Bilder, D. (2017). Organ sculpting by patterned extracellular matrix stiffness. Elife 6, e24958. doi:10.7554 eLife.24958.022

Delon, I. and Brown, N. H. (2009). The integrin adhesion complex changes its composition and function during morphogenesis of an epithelium. J. Cell Sci. 122 4363-4374. doi:10.1242/jcs.055996

Denef, N., Chen, Y., Weeks, S. D., Barcelo, G. and Schüpbach, T. (2008). Crag regulates epithelial architecture and polarized deposition of basement membrane proteins in Drosophila. Dev. Cell 14, 354-364. doi:10.1016/j.devcel.2007.12.012

Devergne, O., Tsung, K., Barcelo, G. and Schüpbach, T. (2014). Polarized deposition of basement membrane proteins depends on Phosphatidylinosito synthase and the levels of Phosphatidylinositol 4,5-bisphosphate. Proc. Natl. Acad. Sci. USA 111, 7689-7694. doi:10.1073/pnas.1407351111

Devergne, O., Sun, G. H. and Schüpbach, T. (2017). Stratum, a homolog of the human GEF Mss4, partnered with Rab8, controls the basal restriction of basement membrane proteins in epithelial cells. Cell Rep 18, 1831-1839. doi:10.1016/j. celrep.2017.02.002

Dzamba, B. J. and DeSimone, D. W. (2018). Extracellular matrix (ECM) and the sculpting of embryonic tissues. Curr. Top. Dev. Biol. 130, 245-274. doi:10.1016/ bs.ctdb.2018.03.006

Frydman, H. M. and Spradling, A. C. (2001). The receptor-like tyrosine phosphatase lar is required for epithelial planar polarity and for axis determination within drosophila ovarian follicles. Development 128, 3209-3220.

Garbincius, J. F. and Michele, D. E. (2015). Dystrophin-glycoprotein complex regulates muscle nitric oxide production through mechanoregulation of AMPK signaling. Proc. Natl. Acad. Sci. USA 112, 13663-13668. doi:10.1073/pnas. 1512991112

Gutzeit, H. O. (1990). The microfilament pattern in the somatic follicle cells of midvitellogenic ovarian follicles of Drosophila. Eur. J. Cell Biol. 53, 349-356.

Gutzeit, H. O., Eberhardt, W. and Gratwohl, E. (1991). Laminin and basement membrane-associated microfilaments in wild-type and mutant Drosophila ovarian follicles. J. Cell Sci. 100, 781-788.

Haigo, S. L. and Bilder, D. (2011). Global tissue revolutions in a morphogenetic movement controlling elongation. Science 331, 1071-1074. doi:10.1126/science. 1199424

Hale, R. and Strutt, D. (2015). Conservation of planar polarity pathway function across the animal kingdom. Annu. Rev. Genet. 49, 529-551. doi:10.1146/ annurev-genet-112414-055224

He, L., Wang, X., Tang, H. L. and Montell, D. J. (2010). Tissue elongation requires oscillating contractions of a basal actomyosin network. Nat. Cell Biol. 12 1133-1142. doi:10.1038/ncb2124

Henry, M. D. and Campbell, K. P. (1998). A role for dystroglycan in basement membrane assembly. Cell 95, 859-870. doi:10.1016/S0092-8674(00)81708-0

Isabella, A. J. and Horne-Badovinac, S. (2015a). Building from the ground up: basement membranes in Drosophila development. Curr. Top. Membr. 76 305-336. doi:10.1016/bs.ctm.2015.07.001

Isabella, A. J. and Horne-Badovinac, S. (2015b). Dynamic regulation of basement membrane protein levels promotes egg chamber elongation in Drosophila. Dev. Biol. 406, 212-221. doi:10.1016/j.ydbio.2015.08.018

Isabella, A. J. and Horne-Badovinac, S. (2016). Rab10-mediated secretion synergizes with tissue movement to build a polarized basement membrane 
architecture for organ morphogenesis. Dev. Cell 38, 47-60. doi:10.1016/j.devcel. 2016.06.009

Lerner, D. W., McCoy, D., Isabella, A. J., Mahowald, A. P., Gerlach, G. F., Chaudhry, T. A. and Horne-Badovinac, S. (2013). A Rab10-dependent mechanism for polarized basement membrane secretion during organ morphogenesis. Dev. Cell 24, 159-168. doi:10.1016/j.devcel.2012.12.005

Morin, X., Daneman, R., Zavortink, M. and Chia, W. (2001). A protein trap strategy to detect GFP-tagged proteins expressed from their endogenous loci in Drosophila. Proc. Natl. Acad. Sci. USA 98, 15050-15055. doi:10.1073/pnas 261408198

Naegeli, K. M., Hastie, E., Garde, A., Wang, Z., Keeley, D. P., Gordon, K. L., Pani, A. M., Kelley, L. C., Morrissey, M. A., Chi, Q. et al. (2017). Cell Invasion In vivo via rapid exocytosis of a transient lysosome-derived membrane domain. Dev. Cell 43, 403-417.e10. doi:10.1016/j.devcel.2017.10.024

Qin, X., Park, B. O., Liu, J., Chen, B., Choesmel-Cadamuro, V., Belguise, K. Heo, W. D. and Wang, X. (2017). Cell-matrix adhesion and cell-cell adhesion differentially control basal myosin oscillation and Drosophila egg chamber elongation. Nat. Commun. 8, 14708. doi:10.1038/ncomms 14708

Sarov, M., Barz, C., Jambor, H., Hein, M. Y., Schmied, C., Suchold, D., Stender, B., Janosch, S., Kj, V. V., Krishnan, R. T. et al. (2016). A genome-wide resource for the analysis of protein localisation in Drosophila. Elife 5, e12068. doi:10.7554/ elife.12068

Satz, J. S., Philp, A. R., Nguyen, H., Kusano, H., Lee, J., Turk, R., Riker, M. J., Hernández, J., Weiss, R. M., Anderson, M. G. et al. (2009). Visual impairment in the absence of dystroglycan. J. Neurosci. 29, 13136-13146. doi:10.1523/ JNEUROSCI.0474-09.2009

Satz, J. S., Ostendorf, A. P., Hou, S., Turner, A., Kusano, H., Lee, J. C., Turk, R. Nguyen, H., Ross-Barta, S. E., Westra, S. et al. (2010). Distinct functions of glial and neuronal dystroglycan in the developing and adult mouse brain. J. Neurosci. 30, 14560-14572. doi:10.1523/JNEUROSCI.3247-10.2010
Sekiguchi, R. and Yamada, K. M. (2018). Basement membranes in development and disease. Curr. Top. Dev. Biol. 130, 143-191. doi:10.1016/bs.ctdb.2018. 02.005

Spradling, A. C., Bate, M. and Arias, A. M. (1993). The development of Drosophila melanogaster. Dev. Drosophila Melanogaster 1, 1-70.

Vachias, C., Fritsch, C., Pouchin, P., Bardot, O. and Mirouse, V. (2014). Tight coordination of growth and differentiation between germline and soma provides robustness for drosophila egg development. Cell Rep 9, 531-541. doi:10.1016/j. celrep.2014.09.035

Venken, K. J. T., Schulze, K. L., Haelterman, N. A., Pan, H., He, Y., Evans-Holm, M., Carlson, J. W., Levis, R. W., Spradling, A. C., Hoskins, R. A. et al. (2011). MiMIC: a highly versatile transposon insertion resource for engineering Drosophila melanogaster genes. Nat. Methods 8, 737-743. doi:10.1038/ nmeth.1662

Viktorinová, I. and Dahmann, C. (2013). Microtubule polarity predicts direction of egg chamber rotation in Drosophila. Curr. Biol. 23, 1472-1477. doi:10.1016/j.cub. 2013.06.014

Viktorinová, I., König, T., Schlichting, K. and Dahmann, C. (2009). The cadherin Fat2 is required for planar cell polarity in the Drosophila ovary. Development 136 4123-4132. doi:10.1242/dev.039099

Wahlström, G., Norokorpi, H.-L. and Heino, T. I. (2006). Drosophila alpha-actinin in ovarian follicle cells is regulated by EGFR and Dpp signalling and required fo cytoskeletal remodelling. Mech. Dev. 123, 801-818. doi:10.1016/j.mod.2006.08. 004

Weir, M. L., Oppizzi, M. L., Henry, M. D., Onishi, A., Campbell, K. P., Bissell, M. J. and Muschler, J. L. (2006). Dystroglycan loss disrupts polarity and beta-casein induction in mammary epithelial cells by perturbing laminin anchoring. J. Cell Sci. 119, 4047-4058. doi:10.1242/jcs.03103

Yatsenko, A. S. and Shcherbata, H. R. (2014). Drosophila miR-9a targets the ECM receptor Dystroglycan to canalize myotendinous junction formation. Dev. Cell $\mathbf{2 8}$ 335-348. doi:10.1016/j.devcel.2014.01.004 